\title{
Improving particle drag predictions in Euler-Lagrange simulations with two-way coupling
}

\author{
Peter J. Ireland ${ }^{1}$ and Olivier Desjardins \\ Sibley School of Mechanical and Aerospace Engineering \\ Cornell University \\ Ithaca, NY 14853
}

\begin{abstract}
Euler-Lagrange methods are popular approaches for simulating particle-laden flows. While such approaches have been rigorously verified in the dilute limit (where particles do not noticeably alter their carrier flow), much less verification has been attempted for cases where the coupling between the two phases leads to non-negligible modifications in the local fluid velocity. We review one of these techniques for coupled fluid-particle flows, the volume-filtered Euler-Lagrange method, and show that it (like many similar methods) provides erroneous predictions for the interphase drag force due to the presence of the particles. We show that these errors are tied to inaccuracies in the numerical implementation of the drag model for systems with two-way coupling. We therefore introduce a simple approach to correct the implementation of this drag model, and show that this corrected implementation provides accurate and grid-independent predictions of particle settling in two-way coupled flows at low particle Reynolds numbers. Finally, we study the effect of the corrected implementation on a more complicated, cluster-induced turbulence flow.
\end{abstract}

Keywords: Euler-Lagrange methods, particle-laden flows, two-way coupling

\section{Introduction}

Euler-Lagrange (E-L) schemes are one of the most common approaches for simulating particle-laden flows. In E-L schemes, the underlying flow field is treated as a continuum and solved on an Eulerian mesh, while particles are treated as discrete points that move based on interactions with the flow field and with each other. Most investigations with E-L schemes have considered incompressible flows where the governing fluid-phase NavierStokes equations have been solved in either pseudospectral $[1,2,3]$ or finite difference $[4,5]$ frameworks. The forces on the particles are generally determined through some simplification of Newton's second law of motion (e.g., $[6,7,8]$ ) with any forces (or torques) acting on the center of mass of the particles.

Some of the first E-L simulations (e.g., $[9,10,11])$ considered particles that were present at sufficiently low mass and volume loadings that their effect on the carrier flow and their interactions with each other could be neglected. These "one-way coupled" simulations have

\footnotetext{
${ }^{1}$ Email address for correspondence: pji22@cornell.edu
} 
provided insight into particle clustering [12], relative velocities [13], and settling speeds [14] in turbulence, droplet growth in atmospheric clouds [15], and plankton distributions in the ocean [16]. The numerical methods for these simulations have been well-tested and adapted over the past three decades, and much insight has been gained with regard to accurate schemes for determining the fluid drag on the particles [17], for simplifying the particle equation of motion [18], and for temporally integrating the particle equation of motion [19].

In many realistic situations, however, the particle mass and volume loadings are such that the particles transfer significant momentum to their underlying carrier fluid. Simulations that represent the effect of the particles on the underlying flow are referred to as either "two-way coupled" (i.e., the particles alter the carrier fluid but do not interact with each other) or "four-way coupled" (i.e., the particles alter the carrier fluid and also interact with each other). In some cases (e.g., turbulence modulation by inertial particles; see [20]), the momentum exchange from the particles to the fluid has a second-order effect, causing slight modifications to the turbulent kinetic energy and dissipation spectra. In other cases (e.g., fluidized bed reactors; see [21]), however, momentum exchange between the particles and the fluid is a leading-order effect that produces most of the velocity fluctuations within the carrier phase. To better understand the underlying physical processes in flows where momentum exchange from the particles to the fluid dominates the system dynamics, $[22,23]$ have recently proposed a new fundamental flow configuration, cluster-induced turbulence (CIT), where heavy particles settling under gravity induce velocity fluctuations in an initially quiescent fluid, which in turn lead to the formation of large-scale particle clusters and significant uncorrelated particle motion.

While many studies over the past two decades have considered both two-way and four-way coupled systems, the underlying numerical methods for these cases are much less rigorous and well-verified than are the corresponding numerical methods for one-way coupled systems. For example, for two-way and four-way coupled systems, [24, 25] showed that standard EulerLagrange methods fail to provide grid-independent predictions for the interphase momentum exchange, while $[25,26]$ found (somewhat counterintuitively) that for typical simulation approaches and low particle Reynolds numbers, drag predictions tend to become less accurate as the grid resolution improves. [26] also noted that lower-order interpolation methods tend to provide better predictions for the drag than the more expensive, high-order schemes recommended in $[27,17]$.

The "force coupling method" (FCM) [28, 29] is notable in that it provides a more rigorous approach for simulating particle-laden flows. Conceptually speaking, the FCM is more similar to an immersed boundary method than to an Euler-Lagrange method, since particle velocities in the FCM are determined by integrating the fluid velocities in the neighborhood of the particle. [28] showed that by appropriately weighting the fluid velocities in the vicinity of a particle, the exact particle drag force can be recovered in coupled fluid-particle flows. However, the FCM generally requires at least 5 to 6 grid points across the particle diameter [29], limiting the total numbers of particles and the domain sizes that can be simulated. The largest FCM simulations to date have therefore been restricted to around 5,000 particles $[30,31]$. The FCM also experiences numerical instabilities when simulating systems where the ratio between the particle and fluid densities exceeds two [29]. Since we are usually interested in gas-liquid or gas-solid suspensions with millions of particles and density ratios of order 1000, the FCM is not suitable for our purposes. 
The overall goal of this paper therefore is to test and improve simulation predictions for the drag force on particles in two-way and four-way coupled systems. In $\S 2$, we will summarize some of the numerical methods used for E-L simulations. In $\S 3$, we will show that for realistic grid spacings, Euler-Lagrange simulations generally do not yield the correct (analytical) result for the drag forces on the particles. In $\S 4$, we will therefore formulate an approach for improving the microscale drag predictions within the volume-filtered EulerLagrange framework of [32]. This approach will be tested in $\S 5$. In $\S 6$, we will study the effect of these improved drag predictions in a CIT simulation. We will close in $\S 7$ by discussing practical implications of this work for two-way and four-way coupled E-L simulations.

\section{Numerical methods for Euler-Lagrange simulations}

In particle-laden flows, particles induce fluid velocity disturbances whose initial length scales are on the order of the particle size. E-L schemes, however, typically use grid spacings that are larger than the particles, and thus are unable to resolve these fluid velocity disturbances explicitly. For very dilute flows, these disturbances are assumed not to influence the overall flow field, and thus no special treatment is needed. However, when the particle mass loading becomes non-negligible, some approach is required to model the effect of these unresolved disturbances. In this section, we discuss the numerical methods used in E-L simulations to account for these unresolved disturbances. We subdivide these methods into those used for traditional E-L simulations (discussed in \$2.1) and those used for volumefiltered E-L simulations (discussed in $\S 2.2$ ). We emphasize that despite apparent differences between the two simulation approaches, they have much in common. In $\S 2.3$, we therefore discuss how some simple modifications can be made to traditional E-L simulations to cast them within the volume-filtered framework.

\subsection{Traditional Euler-Lagrange methods}

Traditional E-L schemes (e.g., see $[20,33,26])$ assume that the drag between the particles and the fluid can be treated as a momentum source in the Navier-Stokes equations. The continuity and momentum equations in this case are

$$
\frac{\partial \rho_{f}}{\partial t}+\nabla \cdot\left(\rho_{f} \boldsymbol{u}_{\boldsymbol{f}}\right)=0
$$

and

$$
\frac{\partial}{\partial t}\left(\rho_{f} \boldsymbol{u}_{\boldsymbol{f}}\right)+\nabla \cdot\left(\rho_{f} \boldsymbol{u}_{\boldsymbol{f}} \otimes \boldsymbol{u}_{\boldsymbol{f}}\right)=\nabla \cdot \boldsymbol{\tau}+\rho_{f} \boldsymbol{g}-\mathfrak{F}^{\text {inter }},
$$

where $\rho_{f}$ is the fluid-phase density, $\boldsymbol{u}_{\boldsymbol{f}}$ is the fluid-phase velocity, $\boldsymbol{g}$ is the gravitational acceleration,

$$
\boldsymbol{\tau} \equiv-p \boldsymbol{I}+\mu_{f}\left[\nabla \boldsymbol{u}_{f}+\left(\nabla \boldsymbol{u}_{f}\right)^{\top}-\frac{2}{3}\left(\nabla \cdot \boldsymbol{u}_{\boldsymbol{f}}\right) \boldsymbol{I}\right],
$$

$p$ is the pressure, $\mu_{f}$ is the fluid-phase dynamic viscosity, and $\boldsymbol{I}$ is the identity tensor. $\mathfrak{F}^{\text {inter }}$ is the force due to interphase coupling, and is written as

$$
\mathfrak{F}^{\text {inter }}(\boldsymbol{x}) \equiv \sum_{n=1}^{N_{p}} \mathcal{P}\left[\delta\left(\boldsymbol{x}_{\boldsymbol{p}}{ }^{(n)}-\boldsymbol{x}\right)\right] \boldsymbol{F}_{\mathrm{drag}}^{(n)},
$$


where $N_{p}$ is the total number of particles and $\mathcal{P}\left[\delta\left(\boldsymbol{x}_{\boldsymbol{p}}{ }^{(n)}-\boldsymbol{x}\right)\right]$ is the numerical projection of the Dirac delta function from the particle center $\boldsymbol{x}_{\boldsymbol{p}}{ }^{(n)}$ to the Eulerian grid point $\boldsymbol{x}$. In most traditional E-L schemes, $\mathcal{P}\left[\delta\left(\boldsymbol{x}_{\boldsymbol{p}}{ }^{(n)}-\boldsymbol{x}\right)\right]$ is computed from the weights used in an extrapolation approach. $\boldsymbol{F}_{\text {drag }}^{(n)}$ is the drag force on a particle, typically modeled as

$$
\boldsymbol{F}_{\text {drag }}^{(n)}=m_{p}^{(n)} f_{D}\left(R e_{p}\right) \frac{\boldsymbol{u}_{\boldsymbol{f}}\left(\boldsymbol{x}_{\boldsymbol{p}}^{(n)}(t), t\right)-\boldsymbol{v}_{\boldsymbol{p}}^{(n)}(t)}{\tau_{p}}+\boldsymbol{F}_{\text {buoyancy }}^{(n)},
$$

where $m_{p}^{(n)}$ is the mass of particle $n, \boldsymbol{v}_{\boldsymbol{p}}{ }^{(n)}$ is the velocity of the particle, $\boldsymbol{F}_{\text {buoyancy }}^{(n)}$ is the buoyant force on the particle, $\tau_{p} \equiv \rho_{p} d_{p}^{2} /\left(18 \mu_{f}\right)$ is the particle response time, $\rho_{p}$ is the particle density, and $d_{p}$ is the particle diameter. $f_{D}$ is a coefficient that in general depends on the particle Reynolds number $R e_{p}$. (Note that for Stokes drag $f_{D}=1$.) $R e_{p}$ is given as

$$
R e_{p} \equiv \frac{\rho_{f}\left|\boldsymbol{u}_{\boldsymbol{f}}\left(\boldsymbol{x}_{\boldsymbol{p}}(t), t\right)-\boldsymbol{v}_{\boldsymbol{p}}(t)\right| d_{p}}{\mu_{f}}
$$

Correlations for $f_{D}$ for various particle Reynolds numbers are provided in $[34,35]$. Particles are advanced by solving

$$
m_{p}^{(n)} \frac{d \boldsymbol{v}_{\boldsymbol{p}}^{(n)}}{d t}=\boldsymbol{F}_{\text {drag }}^{(n)}+\boldsymbol{F}_{\text {collision }}^{(n)}+\boldsymbol{F}_{\text {body }}^{(n)},
$$

where $\boldsymbol{F}_{\text {collision }}^{(n)}$ is the collision force acting on particle $n$ and $\boldsymbol{F}_{\text {body }}^{(n)}$ is the body force acting on the particle (e.g., due to gravity).

There are two crucial drawbacks to the traditional E-L formulation, however. First, (1) and (2) are only strictly valid in the limit of vanishing particle concentration. If this condition is not met, the inertial and viscous flux terms need to be modified to account for volumetric obstructions of the flow due to the presence of particles. Second, as noted in [24], traditional Euler-Lagrange methods fail to provide grid-independent results, since the interphase coupling term contains a grid-dependent projection operator $\mathcal{P}\left[\delta\left(\boldsymbol{x}_{\boldsymbol{p}}{ }^{(n)}-\boldsymbol{x}\right)\right]$. Below, we will show that the volume-filtered Euler-Lagrange method provides a rigorous mathematical framework that enables grid-independent simulations of systems with arbitrary particle concentrations.

\subsection{Volume-filtered Euler-Lagrange method}

The volume-filtered Euler-Lagrange (hereafter 'VFEL') approach applies volume-filtering to all terms in the fluid-phase governing equations to explicitly account for the filtering that occurs as unresolved, particle-scale velocity disturbances are represented on an Eulerian mesh. A detailed formulation for the VFEL approach was developed in [32] based on the work of [36]. We summarize the governing equations and numerical methods below.

The starting point in developing the VFEL approach is the continuity and momentum equations for an incompressible flow given in (1) and (2) (but with the interphase coupling term omitted). A Gaussian filter function $\mathcal{G}$, defined as

$$
\mathcal{G}(r) \equiv\left(2 \pi \sigma_{c}^{2}\right)^{-3 / 2} \exp \left[-r^{2} /\left(2 \sigma_{c}^{2}\right)\right]
$$


is then introduced, where $\sigma_{c}$ is the standard deviation of the Gaussian filter and $r$ is the distance from the point in question to the peak of the Gaussian function. We can also classify the filter width by the full width at half maximum $\delta_{f}$, defined as

$$
\delta_{f} \equiv 2 \sigma_{c} \sqrt{2 \ln 2} .
$$

This filtering approach is then used to define the fluid volume fraction $\alpha_{f}$ as

$$
\alpha_{f}(\boldsymbol{x}, t) \equiv \int_{\mathcal{V}_{f}} \mathcal{G}(|\boldsymbol{x}-\boldsymbol{y}|) d \boldsymbol{y},
$$

where $\mathcal{V}_{f}$ indicates that the integral is taken over all points $\boldsymbol{y}$ occupied by the fluid.

The filter can be applied to any point property of the fluid of arbitrary tensorial order (here denoted as $\boldsymbol{a}(\boldsymbol{x}, t)$ ) by taking

$$
\alpha_{f} \overline{\boldsymbol{a}}(\boldsymbol{x}, t)=\int_{\mathcal{V}_{f}} \boldsymbol{a}(\boldsymbol{y}, t) \mathcal{G}(|\boldsymbol{x}-\boldsymbol{y}|) d \boldsymbol{y} .
$$

where $\overline{\boldsymbol{a}}(\boldsymbol{x}, t)$ denotes the volume-filtered field. The residual component $\boldsymbol{a}^{\prime}(\boldsymbol{x}, t)$ is given by

$$
\boldsymbol{a}^{\prime}(\boldsymbol{x}, t)=\boldsymbol{a}(\boldsymbol{x}, t)-\overline{\boldsymbol{a}}(\boldsymbol{x}, t) .
$$

Following [36], [32] multiply the fluid continuity and momentum equations by $\mathcal{G}$ and integrate over $\mathcal{V}_{f}$. After a number of simplifications, they arrive at the following volumefiltered conservation of mass equation (which we have simplified by assuming that $\rho_{f}$ and $\rho_{p}$ are constant):

$$
\frac{\partial}{\partial t}\left(\alpha_{f} \rho_{f}\right)+\nabla \cdot\left(\alpha_{f} \rho_{f} \overline{\boldsymbol{u}_{\boldsymbol{f}}}\right)=0 .
$$

Note that (13) accounts for temporal variations in the local fluid volume fraction and fluxes of fluid volume fraction, which both result from the transport of particles.

We now consider the volume-filtered form of the fluid momentum equation. In deriving the volume-filtered equation, [32] make a number of simplifying assumptions, which are explained in detail in [36]. Essentially, they require that $\delta_{f} \gg d_{p}$, such that the filtered quantities and the Gaussian filter function vary little over the scale of the particle. They also require that the fluctuation quantities be smoothly varying and that the filtered fluctuation terms be small. As before, we also assume that each phase has a constant density, which gives us

$$
\frac{\partial}{\partial t}\left(\alpha_{f} \rho_{f} \overline{\boldsymbol{u}_{\boldsymbol{f}}}\right)+\nabla \cdot\left(\alpha_{f} \rho_{f} \overline{\boldsymbol{u}_{\boldsymbol{f}}} \otimes \overline{\boldsymbol{u}_{\boldsymbol{f}}}\right)=\nabla \cdot \overline{\boldsymbol{\tau}}+\alpha_{f} \rho_{f} \boldsymbol{g}-\mathcal{F}_{\boldsymbol{u}}+\mathcal{F}_{\boldsymbol{\mu}}-\mathcal{F}^{\text {inter }},
$$

where

$$
\bar{\tau} \equiv-\bar{p} \boldsymbol{I}+\mu_{f}\left[\nabla \overline{\boldsymbol{u}_{f}}+\nabla \overline{\boldsymbol{u}_{\boldsymbol{f}}} \boldsymbol{\top}-\frac{2}{3}\left(\nabla \cdot \overline{\boldsymbol{u}_{\boldsymbol{f}}}\right) \boldsymbol{I}\right] .
$$

$\mathcal{F}_{\boldsymbol{u}}$, given by

$$
\mathcal{F}_{u} \equiv \rho_{f} \nabla \cdot\left(\alpha_{f} \overline{\boldsymbol{u}_{f}^{\prime} \otimes \boldsymbol{u}_{f}^{\prime}}\right)
$$


denotes the residual Reynolds-stress term (which is generally either neglected or modeled using a Reynolds-stress closure), and $\mathcal{F}_{\boldsymbol{\mu}}$, given by

$$
\begin{aligned}
\mathcal{F}_{\boldsymbol{\mu}} \equiv & \nabla \cdot\left[\alpha_{f} \mu_{f}\left(\overline{\nabla \boldsymbol{u}_{\boldsymbol{f}}^{\prime}}\right)\right]-\nabla \cdot\left[\alpha_{f} \mu_{f}\left(\nabla \overline{\boldsymbol{u}_{\boldsymbol{f}}}\right)^{\prime}\right] \\
& +\nabla \cdot\left[\alpha_{f} \mu_{f} \overline{\left(\nabla \boldsymbol{u}_{f^{\prime}}\right)^{\top}}\right]-\nabla \cdot\left[\alpha_{f} \mu_{f}\left(\nabla \overline{\boldsymbol{u}_{\boldsymbol{f}}}\right)^{\boldsymbol{T}^{\prime}}\right] \\
& -\frac{2}{3} \nabla \cdot\left[\alpha_{f} \mu_{f} \overline{\left(\nabla \cdot \boldsymbol{u}_{\boldsymbol{f}}^{\prime}\right)} \boldsymbol{I}\right]+\frac{2}{3} \nabla \cdot\left[\alpha_{f} \mu_{f}\left(\nabla \cdot \overline{\boldsymbol{u}_{\boldsymbol{f}}}\right)^{\prime} \boldsymbol{I}\right]
\end{aligned}
$$

is the residual viscous stress (which can be modeled using a volume-fraction-dependent viscosity; e.g., see [37]).

The interphase momentum exchange in (14) is given by $\mathcal{F}^{\text {inter }}$ and represents the effect of the particles on the underlying fluid. It can be subdivided into a resolved contribution $\mathcal{F}_{\text {resolved }}^{\text {inter }}$ and an residual (unresolved) contribution $\mathcal{F}_{\text {residual }}^{\text {inter }}$

$$
\mathcal{F}^{\text {inter }}=\mathcal{F}_{\text {resolved }}^{\text {inter }}+\mathcal{F}_{\text {residual }}^{\text {inter }}
$$

The resolved contribution is given as

$$
\mathcal{F}_{\text {resolved }}^{\text {inter }}=\sum_{n=1}^{N_{p}} \mathcal{G}\left(\left|\boldsymbol{x}-\boldsymbol{x}_{p}^{(n)}\right|\right) \boldsymbol{F}_{\text {resolved }}^{(n)},
$$

where

$$
\boldsymbol{F}_{\text {resolved }}^{(n)}=\int_{\mathcal{S}^{(n)}} \boldsymbol{n} \cdot \overline{\boldsymbol{\tau}} d \boldsymbol{y}
$$

The integration limit $\mathcal{S}^{(n)}$ denotes that the integration is performed over the surface of particle $n$. Under the conditions given above, we can simplify $\boldsymbol{F}_{\text {resolved }}^{(n)}$ to obtain

$$
\boldsymbol{F}_{\text {resolved }}^{(n)} \approx \mathcal{V}_{p}^{(n)} \nabla \cdot \overline{\boldsymbol{\tau}}
$$

where $\mathcal{V}_{p}^{(n)}$ is the volume occupied by particle $n$. We can use this simplification to re-write (19) as

$$
\mathcal{F}_{\text {resolved }}^{\text {inter }}=\left(1-\alpha_{f}\right) \nabla \cdot \overline{\boldsymbol{\tau}}
$$

The residual contribution is given by

$$
\mathcal{F}_{\text {residual }}^{\text {inter }}=\sum_{n=1}^{N_{p}} \mathcal{G}\left(\left|\boldsymbol{x}-\boldsymbol{x}_{p}^{(n)}\right|\right) \boldsymbol{F}_{\text {residual }}^{(n)}
$$

where

$$
\boldsymbol{F}_{\text {residual }}^{(n)}=\int_{\mathcal{S}^{(n)}} \boldsymbol{n} \cdot\left\{-p^{\prime} \boldsymbol{I}+\mu_{f} \nabla \boldsymbol{u}_{\boldsymbol{f}}^{\prime}+\mu_{f}\left(\nabla \boldsymbol{u}_{\boldsymbol{f}}^{\prime}\right)^{\top}-\frac{2}{3} \mu_{f}\left(\nabla \cdot \boldsymbol{u}_{\boldsymbol{f}}^{\prime}\right) \boldsymbol{I}\right\} d \boldsymbol{y}
$$

$\boldsymbol{F}_{\text {residual }}^{(n)}$ is not computable in the VFEL approach and must be modeled. In [32], a simple model is used for $\boldsymbol{F}_{\text {residual }}^{(n)}$, giving

$$
\boldsymbol{F}_{\text {residual }}^{(n)}(t)=m_{p}^{(n)} \alpha_{f}\left(\boldsymbol{x}_{\boldsymbol{p}}^{(n)}(t), t\right) f_{D}\left(\alpha_{f}, R e_{p}\right) \frac{\overline{\boldsymbol{u}_{\boldsymbol{f}}}\left(\boldsymbol{x}_{\boldsymbol{p}}{ }^{(n)}(t), t\right)-\boldsymbol{v}_{\boldsymbol{p}}{ }^{(n)}(t)}{\tau_{p}} .
$$


Lagrangian particles are evolved by solving a simplified form of Newton's second law for each particle,

$$
m_{p}^{(n)} \frac{d \boldsymbol{v}_{\boldsymbol{p}}^{(n)}}{d t}=\boldsymbol{F}_{\text {resolved }}^{(n)}+\boldsymbol{F}_{\text {residual }}^{(n)}+\boldsymbol{F}_{\text {collision }}^{(n)}+\boldsymbol{F}_{\text {body }}^{(n)}
$$

We now highlight some advantages of the VFEL approach, as compared to traditional E-L methods. First, the governing equations for the VFEL approach are derived from first principles, and (unlike traditional Euler-Lagrange methods) are valid for arbitrary particle

loadings (provided that an accurate model is given for $\boldsymbol{F}_{\text {residual }}^{(n)}$ ). Second, as discussed in [32], the VFEL approach enables grid-independent solutions for two-way coupled systems. Third, by decomposing the interphase coupling term into resolved and residual components, the VFEL method can capture explicitly the effect of turbulent lengthscales larger than the particle diameter. For example, $[22,23]$ showed that the VFEL method is able to resolve the influence of particle clusters on the surrounding fluid, which in turn leads to the ability to distinguish between spatially correlated and uncorrelated particle motion. In Appendix A and Appendix B, we will determine the extent to which large-scale features are resolved in the VFEL approach in several canonical flows. Fourth, a clear distinction is made in the VFEL between fluid velocities and particle velocities, and the local fluid volume fraction field $\alpha_{f}$ is calculated. When computing fluid-phase statistics, the quantities can be weighted appropriately based on the local value of $\alpha_{f}$, giving accurate fluid-phase and particle-phase statistics for arbitrarily low $\alpha_{f}$.

\subsection{Adapting traditional Euler-Lagrange methods to a volume-filtered framework}

Given the advantages of the VFEL approach to the traditional E-L approach, it is useful to describe the simple changes needed to adapt a traditional E-L code a VFEL code. In comparing (1) and (2) with (13) and (14), respectively, we observe that the former two equations contain point-wise (for finite difference approaches) or cell-averaged (for finite volume approaches) values of flow variables, while the latter two equations contain volumefiltered values. The first step in implementing the VFEL approach is therefore to introduce an operator to apply volume-filtering to the flow quantities. A simple and computationally efficient way to do so is through the implicit diffusion approach discussed in [32].

If the unclosed terms, $\mathcal{F}_{\boldsymbol{u}}$ and $\mathcal{F}_{\boldsymbol{\mu}}$, are neglected, the only other differences between the two approaches are the inclusion of the fluid volume fraction in some of the terms for the VFEL method and the treatment of the interphase coupling term. The first issue requires only a simple modification to the governing equations. We therefore focus on the second issue, the treatment of the interphase coupling term.

In comparing (4) and (18), we notice four differences. First, (18) includes the effect of resolved stresses, which are omitted in (4). These stresses can be easily included through (22). Second, buoyancy forces are omitted in (18), while they are included in (4). In Appendix A.2, we demonstrate that buoyancy forces are captured entirely within our resolved drag force, and thus do not need to be included separately in the VFEL method. Third, the interphase coupling forces in (4) are computed via a projection operation involving extrapolation, while (18) involves a Gaussian projection. [32] showed that the extrapolation approach can be easily combined with a filtering approach to yield a grid-independent Gaussian projection. Fourth, the particle drag law in the traditional E-L approach given in (5) is slightly altered in the formulation provided in (25). 
These simple modifications will therefore allow a traditional E-L method to be adapated to the more robust and mathematically rigorous VFEL approach. In the next section, we therefore test how accurately we are able to predict particle settling velocities using the VFEL approach.

\section{Predicted particle settling speeds for the VFEL approach}

We now test the accuracy of the VFEL approach for a simple case of a single particle settling in an initially quiescent flow in an unbounded domain. We assume that the particle Reynolds number is sufficiently low that the drag on the particle can be modeled through a simple Stokes drag law, and based on (26), we obtain the analytical prediction for the particle settling velocity to be

$$
\boldsymbol{v}_{\boldsymbol{p}}(t)=U \frac{\boldsymbol{g}}{|\boldsymbol{g}|}\left[1-\exp \left(-t / \tau_{p}\right)\right]
$$

where $U \equiv \tau_{p}|\boldsymbol{g}|\left(1-\rho_{f} / \rho_{p}\right)$ is the magnitude of the gravitational settling velocity at steadystate.

We then use the VFEL approach to perform a simulation of low-Reynolds-number settling in the initially quiescent, triply periodic domain. Gravity is directed in the $x_{1}$-direction. We take the ratio between particle and fluid densities $\rho_{p} / \rho_{f}$ to be 1000 , which is representative of a liquid in air at atmospheric conditions. To ensure that we remain well within the Stokes drag regime, we use a particle Reynolds number $R e_{p}=0.001$. We simulate a domain of size $\left(256 d_{p}\right)^{3}$ to ensure that any disturbance flows disappear far away from the particles and that the simulations are not artificially impacted by the periodic boundary conditions. We consider four different values of $\delta_{f} / d_{p}$ : 2, 4, 8, and 16. In all cases, we choose the grid spacing $\delta x$ (taken to be uniform in all directions) to give $\delta_{f} / \delta x=4$, such that our filter is adequately captured on our grid with a resolution comparable to that in typical published VFEL studies $[22,23,38]$. The fluid quantities at the particle locations are computed using linear interpolation.

Before considering the results, we anticipate the possible outcomes. The first and desired outcome is that the settling velocities in the numerical simulations uniformly match the analytical prediction from (27). The second possible outcome is that the particle settling speeds are under-predicted for certain values of $\delta_{f}$. This would indicate that we are overpredicting the sum of the resolved and residual drag, and thus that our model equation for the residual drag, (25), must be changed to predict less drag for these filter sizes. The third possible outcome is that the particle settling speeds are over-predicted for certain values of $\delta_{f}$. In this case, our model equation for the residual drag, (25), would need to be modified to predict more drag for these filter sizes.

From figure 1, we see that outcome three is occurring: we are under-predicting the drag on the particles, which in turn causes us to over-predict the particle settling velocity. We also notice that the errors in the settling velocity decrease as we increase the filter size. It is important to point out these issues are not unique to the VFEL approach, but are present for coupled E-L simulations in general. For example, [26] also observed erroneous settling predictions when using traditional E-L methods with two-way coupling and noted that the errors in the settling velocity increase as the grid spacing decreases (for a fixed 


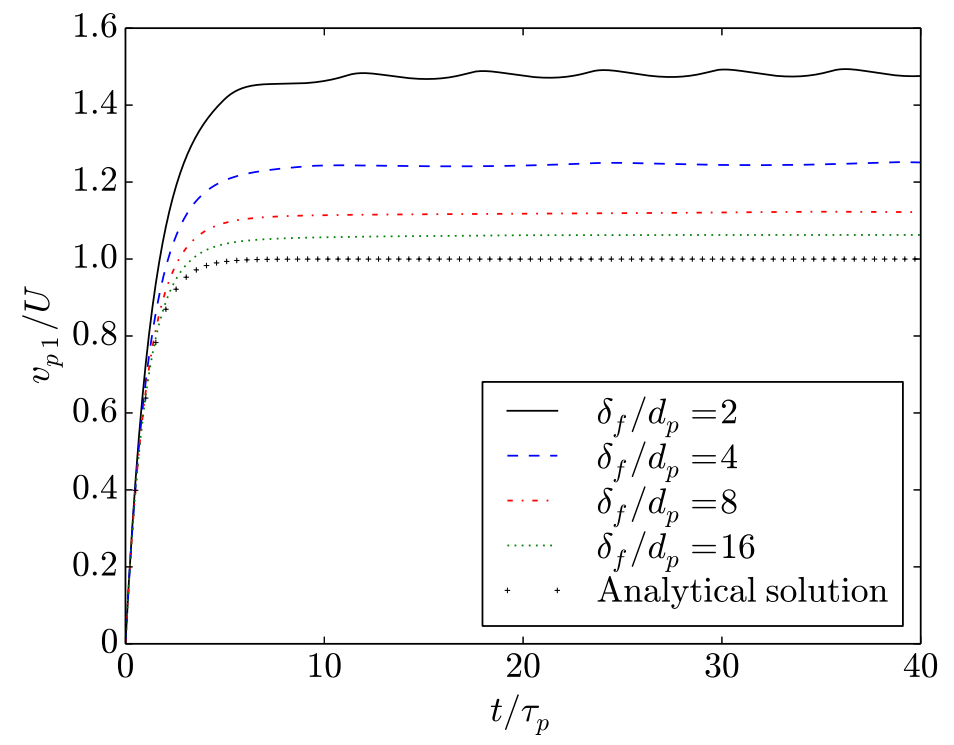

Figure 1: Particle settling speeds obtained using the original VFEL approach described in [32]. The simulations are performed with $R e_{p}=0.001$ and $\delta_{f} / \delta x=4$ for various filter sizes, and the results are compared to the analytical (Stokes) solution.

particle diameter). For the purposes of this paper, our focus will be on improving the VFEL approach to correct these drag predictions. As we will see in $\S 4$, the VFEL approach, with its well-posed governing equations and grid-independent filtering procedure, allows us to derive closed-form analytical expressions to correct the drag on the particles. The same is generally not true for traditional E-L methods, which involve implicit filtering and provide grid-dependent predictions of interphase coupling terms.

\section{Correcting the residual drag predictions}

\subsection{Summary of problem and review of suggested corrections}

In order to understand the errors observed in $\S 3$, it is useful to compare the formulation for the particle drag in the VFEL method to that in a standard drag law, from which the analytical solution can be obtained. Standard particle drag laws assume that the superficial (or "undisturbed") values of the fluid velocity and fluid volume fraction are specified. That is, the fluid velocity and the fluid volume fraction used in a standard drag correlation should be those obtained if the particle in question had no effect on the flow. We will denote the undisturbed fluid volume fraction as $\widetilde{\alpha_{f}}$ and the undisturbed fluid velocity as $\widetilde{\boldsymbol{u}_{\boldsymbol{f}}}$. Using a standard particle drag law, we would have

$$
\boldsymbol{F}_{\text {drag }}=m_{p} \widetilde{\alpha_{f}}\left(\boldsymbol{x}_{\boldsymbol{p}}(t), t\right) f_{D}\left(\widetilde{\alpha_{f}}, R e_{p}\right) \frac{\widetilde{\boldsymbol{u}_{\boldsymbol{f}}}\left(\boldsymbol{x}_{\boldsymbol{p}}(t), t\right)-\boldsymbol{v}_{\boldsymbol{p}}^{(n)}(t)}{\tau_{p}}+\boldsymbol{F}_{\text {buoyancy }}
$$

Within the VFEL framework, we have

$$
\boldsymbol{F}_{\text {drag }}=\boldsymbol{F}_{\text {residual }}+\boldsymbol{F}_{\text {resolved }}
$$


In Appendix A, we demonstrate that for $\delta_{f} / d_{p} \gtrsim 4, \boldsymbol{F}_{\text {resolved }} \rightarrow \boldsymbol{F}_{\text {buoyancy }}$. Using this result and (25), we can write (29) as

$$
\boldsymbol{F}_{\mathrm{drag}} \approx m_{p}^{(n)} \alpha_{f}\left(\boldsymbol{x}_{\boldsymbol{p}}^{(n)}(t), t\right) f_{D}\left(\alpha_{f}, R e_{p}\right) \frac{\overline{\boldsymbol{u}_{\boldsymbol{f}}}\left(\boldsymbol{x}_{\boldsymbol{p}}{ }^{(n)}(t), t\right)-\boldsymbol{v}_{\boldsymbol{p}}^{(n)}(t)}{\tau_{p}}+\boldsymbol{F}_{\text {buoyancy }}
$$

By comparing (28) and (30), we observe that that the only differences between the two relations are in the volume fractions and fluid velocities used in computing the residual drag. A standard particle drag law, (28), uses $\widetilde{\alpha_{f}}$, the undisturbed fluid volume fraction at the particle location, and $\widetilde{\boldsymbol{u}_{\boldsymbol{f}}}$, the undisturbed fluid velocity at the particle location. The model currently used in the VFEL framework, (30), uses $\alpha_{f}$, the filtered fluid volume fraction at the particle location, and $\overline{\boldsymbol{u}_{\boldsymbol{f}}}$, the filtered fluid velocity at the particle location. To improve the simulated particle settling speeds shown in $\S 3$, we therefore seek an approach to estimate $\widetilde{\alpha_{f}}$ and $\widetilde{\boldsymbol{u}_{f}}$ given $\alpha_{f}$ and $\overline{\boldsymbol{u}_{\boldsymbol{f}}}$.

Before doing so, however, it is useful to pause and provide a physical explanation for the differences between the filtered and undisturbed values and the resulting impact on the simulated drag forces on the particles. In a two-way (or four-way) coupled flow, the presence of a particle will lead to a smaller filtered fluid volume fraction $\alpha_{f}$ near the particle. It will also induce a local disturbance in the fluid velocity, which will cause the filtered fluid velocity $\overline{\boldsymbol{u}_{\boldsymbol{f}}}$ near the particle to be closer to the particle velocity $\boldsymbol{v}_{\boldsymbol{p}}$. Therefore, due to the disturbances induced by the particle, $\alpha_{f}\left(\boldsymbol{x}_{\boldsymbol{p}}{ }^{(n)}(t), t\right)<\widetilde{\alpha_{f}}\left(\boldsymbol{x}_{\boldsymbol{p}}{ }^{(n)}(t), t\right)$ and $\left|\overline{\boldsymbol{u}_{\boldsymbol{f}}}\left(\boldsymbol{x}_{\boldsymbol{p}}{ }^{(n)}(t), t\right)-\boldsymbol{v}_{\boldsymbol{p}}{ }^{(n)}(t)\right|<$ $\left|\widetilde{\boldsymbol{u}_{\boldsymbol{f}}}\left(\boldsymbol{x}_{\boldsymbol{p}}{ }^{(n)}(t), t\right)-\boldsymbol{v}_{\boldsymbol{p}}{ }^{(n)}(t)\right|$. The result will be an under-prediction of the drag on a particle and an over-prediction of the particle settling velocity. As the filter size decreases, the effect of the particle on the filtered fluid velocity and the filtered fluid volume fraction will be concentrated near the particle, exacerbating this issue. This explains the counter-intuitive observation in $\S 3$ that the simulated particle settling velocity becomes less accurate as the filter size decreases.

The issue of estimating the undisturbed fluid properties from the disturbed fluid properties for two-way coupled, particle-laden turbulence is addressed in two recent papers [25, 26]. (Note that both studies do not consider the effect of the disturbed volume fraction on the drag and limit their attention to the issue of particle-induced fluid velocities.) In [25], the authors approximate the influence of the particle on the flow as a point-wise force that has diffused over a small time $\epsilon_{R}$. Under this approximation, they compute the disturbance flow generated by the particle, and use an explicit Euler approach to account for the extent to which this disturbance has diffused over the time $\epsilon_{R}$. They can then remove this diffused disturbance velocity from the fluid velocity at the particle location, and can thereby accurately compute the drag acting on the particle through the model equations in [7].

In [26], the authors assume that the influence on the particle on the fluid velocity field near the particle can be approximated by

$$
\widetilde{\boldsymbol{u}_{\boldsymbol{f}}}=\overline{\boldsymbol{u}_{\boldsymbol{f}}}+\mathcal{C} \delta x^{2} \nabla^{2} \overline{\boldsymbol{u}_{\boldsymbol{f}}}
$$

where $\mathcal{C}$ depends on both the ratio between the particle size and the grid spacing, and also the position of the particle on the grid. They then place particles in a flow under various combinations of these parameters, subject the particles to a drag force, and compute and 
tabulate $\mathcal{C}$ for each set of parameters. These tabulated values are then used in (31) to estimate $\widetilde{\boldsymbol{u}_{\boldsymbol{f}}}$.

We would like to develop an analytical correction approach for the VFEL method that does not require prior tabulation of values for a range of different parameters. Our approach for removing particle-induced fluid disturbances is motivated by an analogous problem in droplet vaporization, which is addressed and resolved in [39]. In spray calculations, a pointsource approximation is often used to represent the influence of a small droplet immersed in the gas phase. The evaporation or condensation rate of the droplet is then modeled by solving an equation for the transfer number. This equation, like the analogous expression for the drag given in (28), requires information about the undisturbed fluid properties, which are generally not available in a coupled simulation. [39] showed that simply using the filtered fluid properties causes the computed evaporation or condensation rates to become progressively less accurate as the grid resolution increases. In [39], however, the authors derive an exact relationship for difference between the transfer numbers in the undisturbed and filtered fields for various grid resolutions in the limit of negligible Reynolds number. Using this relationship, they recover the undisturbed transfer number given only the filtered transfer number, allowing for accurate calculations of evaporation or condensation rates for particle diameters on the order of the mesh spacing.

\subsection{Fluid volume fraction correction}

We first consider an approach for obtaining the undisturbed fluid volume fraction $\widetilde{\alpha_{f}}$ from the filtered fluid volume fraction $\alpha_{f}$. We consider a single particle immersed in an infinite fluid. Since (25) requires knowledge of fluid properties at the particle center, we evaluate (10) at $\boldsymbol{x}=\boldsymbol{x}_{\boldsymbol{p}}$, giving

$$
\alpha_{f}=1-\operatorname{erf}\left[\frac{1}{\widehat{\sigma}_{c} \sqrt{2}}\right]+\frac{\sqrt{2 / \pi}}{\widehat{\sigma}_{c}} \exp \left[-\frac{1}{2 \widehat{\sigma}_{c}^{2}}\right],
$$

where $\widehat{\sigma}_{c} \equiv \sigma_{c} / r_{p}=\left(\delta_{f} / d_{p}\right) / \sqrt{2 \ln 2}$.

We know that the undisturbed flow is characterized by $\widetilde{\alpha_{f}}=1$, and thus the difference between the undisturbed and filtered fluid volume fractions is given by

$$
\zeta_{\alpha_{f}} \equiv \widetilde{\alpha_{f}}-\alpha_{f}=\operatorname{erf}\left[\frac{1}{\widehat{\sigma}_{c} \sqrt{2}}\right]-\frac{\sqrt{2 / \pi}}{\widehat{\sigma}_{c}} \exp \left[-\frac{1}{2 \widehat{\sigma}_{c}^{2}}\right]
$$

We can therefore recover the undisturbed fluid volume fraction from the filtered value simply by taking

$$
\widetilde{\alpha_{f}}\left(\boldsymbol{x}_{\boldsymbol{p}}(t), t\right)=\alpha_{f}\left(\boldsymbol{x}_{\boldsymbol{p}}(t), t\right)+\zeta_{\alpha_{f}} .
$$

Note that the only requirements for this correction to be valid are that the domain is unbounded and that the particles are spherical. The volume fraction correction will hold for arbitrary flow configurations over the full range of particle Reynolds numbers.

$\zeta_{\alpha_{f}}$ is plotted in figure 2 for various values of $\delta_{f} / d_{p}$. We notice that $\zeta_{\alpha_{f}}$ decreases with increasing $\delta_{f} / d_{p}$. This indicates that using the filtered fluid volume fraction becomes less problematic as the resolution decreases, which is similar to the observations for the transfer rate in [39] and for the particle drag in [26]. 


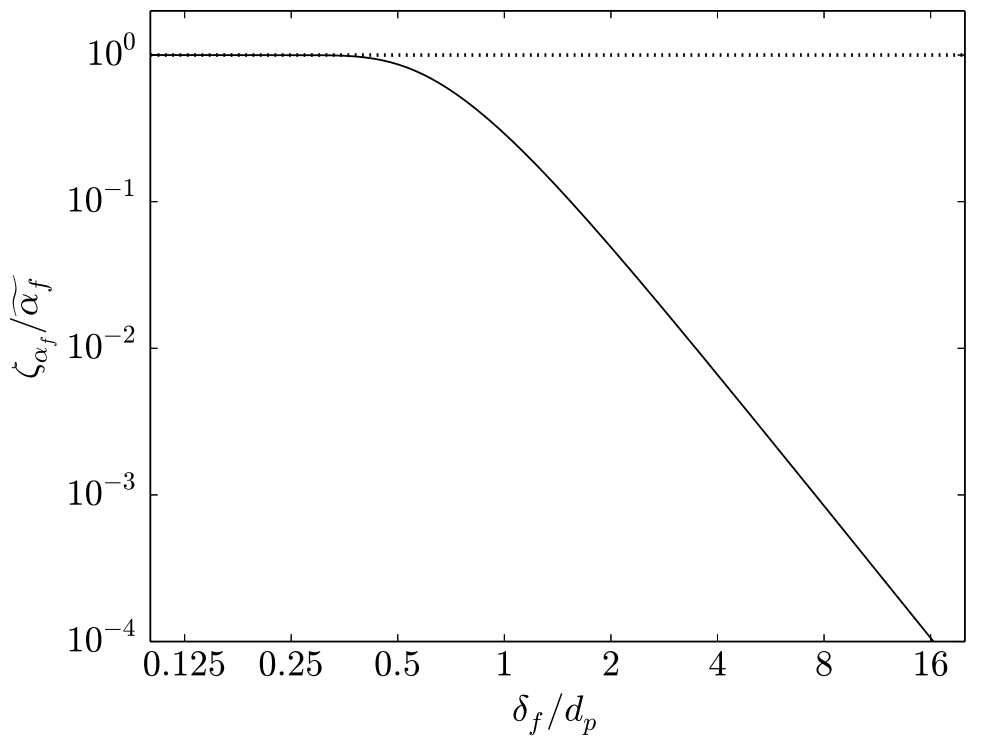

Figure 2: The difference between the undisturbed and disturbed fluid volume fractions for various filter sizes $\delta_{f}$.

\subsection{Fluid velocity correction}

We next introduce a correction to obtain the undisturbed fluid velocity from the filtered value. For finite values of the particle Reynolds number, the interactions between the particles and their disturbance flows are nonlinear, and thus no simple analytical expression is available to recover the undisturbed velocities. At low particle Reynolds numbers (i.e., Stokes flows), however, the problem is linear, and we can analytically compute the disturbance flow. We therefore first consider a Stokes flow around a particle which is moving in the $x_{1}$-direction, and then generalize for an arbitrary orientation of the particle velocity. In $\S 5.4$, we will examine how appropriate our approach is for larger values of $R e_{p}$.

The analytical solution for the steady-state Stokes flow around a particle settling in the $x_{1}$-direction is given in (A1). In the VFEL framework, we solve for the filtered fluid velocity at the particle position, which is defined based on (11) as

$$
\alpha_{f} \overline{\boldsymbol{u}_{\boldsymbol{f}}} \equiv \int_{\mathcal{V}_{f}} \boldsymbol{u}_{\boldsymbol{f}}(\boldsymbol{y}, t) \mathcal{G}(|\boldsymbol{x}-\boldsymbol{y}|) d \boldsymbol{y} .
$$

In general, no closed-form solution for $\boldsymbol{u}_{\boldsymbol{f}}(\boldsymbol{y}, t)$ is available. Assuming a steady-state Stokes solution for the velocity, however, we can evaluate (35) by taking $\boldsymbol{x}=\boldsymbol{x}_{\boldsymbol{p}}$, from which we find that

$$
\begin{aligned}
\frac{\bar{u}_{1}}{U} & =\frac{\frac{\sqrt{2 / \pi}}{\widehat{\sigma_{c}}} \exp \left[-\frac{1}{2{\widehat{\sigma_{c}}}^{2}}\right]}{1-\operatorname{erf}\left[\frac{1}{\widehat{\sigma_{c}} \sqrt{2}}\right]+\frac{\sqrt{2 / \pi}}{\widehat{\sigma_{c}}} \exp \left[-\frac{1}{2 \widehat{\sigma}^{2}}\right]} \equiv \zeta_{u_{f}}, \\
\frac{\bar{u}_{f}}{U} & =0 \\
\frac{\bar{u}_{f}}{U} & =0 .
\end{aligned}
$$


Note that these expressions are not generally valid for particles whose velocities are not described by a steady-state Stokes prediction, as would be the case for highly collisional

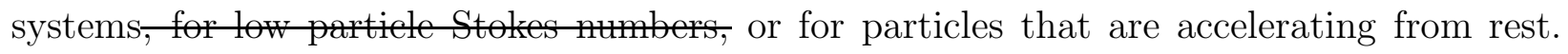
(A similar assumption of steady-state conditions was made in [26], and the authors also provide the same qualifications for transient and collisional particle motion.) These expressions, however, are expected to be accurate for dilute, low-Reynolds-number flows

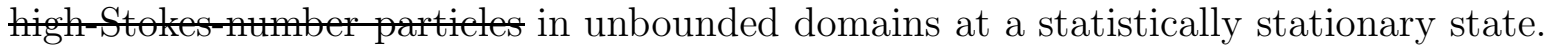

For the case of a single particle settling in an initially quiescent flow, ${\overline{u_{f}}}_{2}$ and ${\overline{u_{f}}}_{3}$ given by (36) are exactly correct. We can calculate $\widetilde{u_{f_{1}}} / U$ by taking

$$
\widetilde{u_{f_{1}}} / U=\frac{\overline{u_{f}}}{U}-\zeta_{u_{f}} \text {. }
$$

We note that $U$ is simply the magnitude of the slip velocity, which at steady-state is equivalent to $v_{p_{1}}-\widetilde{u_{f}}$. Substituting this expression into (37) and simplifying yields

$$
\widetilde{u_{f}}=\frac{\bar{u}_{f}-v_{p_{1}} \zeta_{u_{f}}}{1-\zeta_{u_{f}}} \text {. }
$$

It is straightforward to generalize this result to an arbitrary orientation of the particle velocity, which gives us

$$
\widetilde{\boldsymbol{u}_{\boldsymbol{f}}}=\frac{\overline{\boldsymbol{u}_{\boldsymbol{f}}}-\boldsymbol{v}_{\boldsymbol{p}} \zeta_{u_{f}}}{1-\zeta_{u_{f}}}
$$

We plot $\zeta_{u_{f}}$ at different values of $\delta_{f} / d_{p}$ in figure 3 . We notice that the velocity corrections decay much more slowly than the volume fraction corrections (see figure 2), and are significant even for the filter sizes typically used in the VFEL method. These findings suggest that the correction approach described above is necessary to reduce the errors to an acceptable level.

\subsection{Summary of proposed residual drag corrections}

We now summarize the method proposed for improving the residual drag predictions. The residual drag is computed as

$$
\begin{aligned}
\boldsymbol{F}_{\text {residual }}^{(n)}(t)= & m_{p}\left[\alpha_{f}\left(\boldsymbol{x}_{\boldsymbol{p}}{ }^{(n)}(t), t\right)+\zeta_{\alpha_{f}}\right] f_{D}\left(\alpha_{f}\left(\boldsymbol{x}_{\boldsymbol{p}}{ }^{(n)}(t), t\right)+\zeta_{\alpha_{f}}, R e_{p}\right) \\
& \times\left[\frac{\frac{\overline{\boldsymbol{u}_{\boldsymbol{f}}}\left(\boldsymbol{x}_{\boldsymbol{p}}{ }^{(n)}(t), t\right)-\boldsymbol{v}_{\boldsymbol{p}}{ }^{(n)}(t) \zeta_{u_{f}}}{1-\zeta_{u_{f}}}-\boldsymbol{v}_{\boldsymbol{p}}{ }^{(n)}(t)}{\tau_{p}}\right]
\end{aligned}
$$

where the volume fraction correction $\zeta_{\alpha_{f}}$ is given by (34) and the fluid velocity correction $\zeta_{u_{f}}$ is given by (36). A key advantage of our formulation of the drag corrections, as opposed to the method given by [26], is that our approach is entirely analytical and does not require the use of pre-tabulated, grid-dependent coefficients.

(40) is derived under the assumptions that we have a three-dimensional, unbounded, lowReynolds-number flow of high-Stokes-number particles, that our filter is resolved adequately on our computational domain $\left(\delta_{f} / \delta x \gg 1\right)$, that the particle and fluid velocities are at an approximately statistically stationary state, and that particle collisions are infrequent. In $\S 5$, we will test our proposed corrections to determine how effective they are over a range of filter sizes, grid resolutions, and Reynolds numbers. 


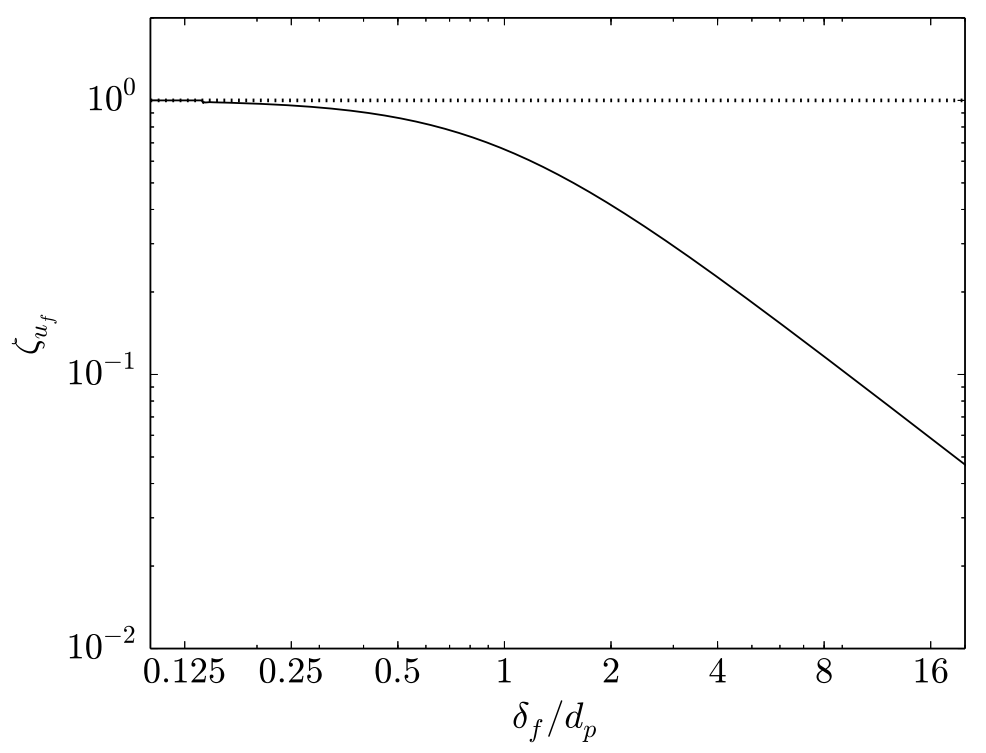

Figure 3: The velocity correction factor $\zeta_{u_{f}}$, plotted for different filter sizes $\delta_{f}$.

\section{Testing the residual drag corrections}

We now test the effectiveness of the fluid volume fraction and fluid velocity corrections proposed in $\S 4.2$ and $\S 4.3$, respectively, for improving the prediction of the residual $\operatorname{drag} \boldsymbol{F}_{\text {residual. }}^{(n)}$ As was done in [26], we can use the Buckingham Pi theorem to form nondimensional groups. For the VFEL method, these groups are taken to be $\delta_{f} / d_{p}$ (the filter size), $\rho_{p} / \rho_{f}$ (the density ratio), and $R e_{p}$ (the particle Reynolds number). Note that a fourth group is also possible: $\delta_{f} / \delta x$, which represents the filter resolution. However, the VFEL approach is designed to give grid-independent results for a sufficiently resolved filter $\delta_{f}$. We therefore expect that our results should be independent of $\delta_{f} / \delta x$ for $\delta_{f} / \delta x \gg 1$. We test the effect of each of these non-dimensional parameters below.

Finally, since our correction scheme was devised under the assumption of steady-state conditions, it is also useful to quantify the effects of unsteadiness on the simulated settling speeds. To do so, we will follow the approach of [26] by introducing a time-dependent bodyforce to $(26)$,

$$
\boldsymbol{F}_{\text {body }}^{(n)}=m_{p}^{(n)} \boldsymbol{g} \sin \left(t / \tau_{\text {body }}\right),
$$

where $\tau_{\text {body }}$ is the characteristic timescale of the bodyforce. From this, we can formulate a bodyforce Stokes number, $S t_{\text {body }} \equiv \tau_{p} / \tau_{\text {body }}$. We will also assess how our results are affected by variations in $S t_{\text {body }}$ below.

\subsection{Effect of the filter size}

To test the effect of the filter size, $\delta_{f} / d_{p}$, on our drag corrections, we repeat the simulations in $\S 3$, but now with the residual drag computed via (40). To decouple the effects of the volume fraction and velocity corrections, we also performed simulations with just the volume fraction correction (i.e., computing the residual drag via (40) but taking $\zeta_{u_{f}} \equiv 0$ ) and with just the fluid velocity correction (i.e., computing the residual drag via (40) but taking $\zeta_{\epsilon_{f}} \equiv 0$ ). As in $\S 3$, the simulations are performed using $\rho_{p} / \rho_{f}=1000, R e_{p}=0.001$, a domain size of $\left(256 d_{p}\right)^{3}$, 

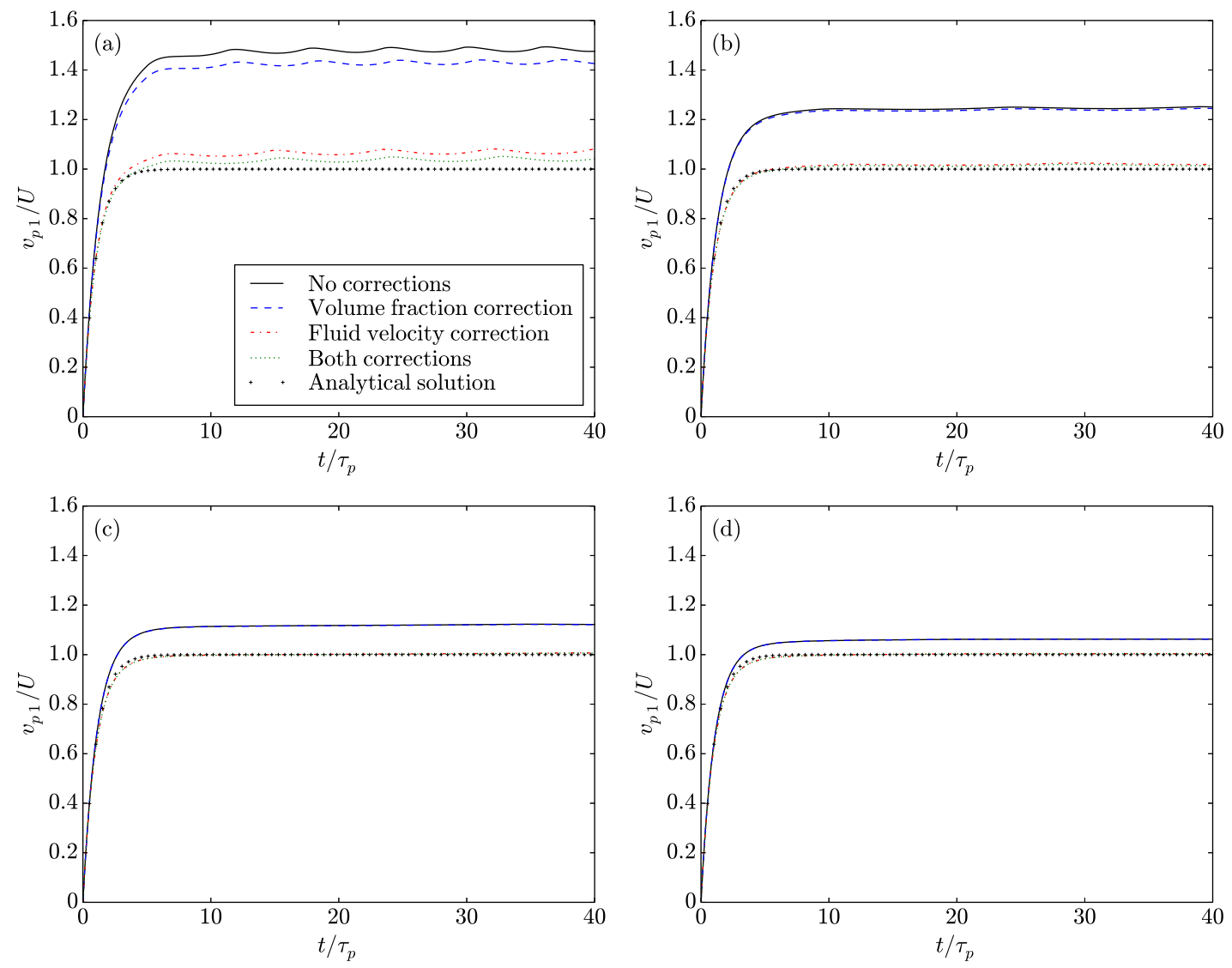

Figure 4: Particle settling speeds with and without the drag corrections from $\S 4$ for filter sizes $\delta_{f} / d_{p}=2$ (a), $\delta_{f} / d_{p}=4(\mathrm{~b}), \delta_{f} / d_{p}=8(\mathrm{c})$, and $\delta_{f} / d_{p}=16$ (d). The simulations are performed with $R e_{p}=0.001$, $\delta_{f} / \delta x=4$, and $\rho_{p} / \rho_{f}=1000$ for various filter sizes, and the results are compared to the analytical (Stokes) solution.

and a grid resolution $\delta_{f} / \delta x=4$. The results for four different filter sizes $\delta_{f}$ are given in figure 4 , and results from $\S 3$ (i.e., with neither the volume fraction nor velocity corrections) are included for comparison. The root-mean-square errors in $v_{p_{1}} / U$ (for $0 \leq t / \tau_{p} \leq 100$ ) are also calculated, and are shown in table 1.

We notice that when both corrections are applied, the error in the particle settling velocity is reduced, yielding results that are close to the analytical predictions. In general, we notice that the volume fraction correction has a weak or negligible effect on the predicted settling speed, while the fluid velocity correction has a significant effect in all cases. Finally, we observe that small errors in the settling velocity persist for $\delta_{f} / d_{p}=2$ and $\delta_{f} / d_{p}=4$, even when both corrections are applied; for larger filter sizes, however, the corrections are able to almost completely eliminate any errors, presumably because the underlying assumptions of the VFEL framework are better satisfied in these cases (see §2.2).

Note that similar results were obtained in both [25] and [26]. [25] showed that the settling velocity errors increase as the particle becomes large with respect to the filter size, while [26] noted that large errors were seen as the particle diameter approached the grid spacing. Both studies were able to reduce the errors by applying their respective correction approaches. 
Table 1: Percent root-mean-square error in $v_{p_{1}} / U$ for various filter sizes and with various approaches for computing the residual drag. All simulations are performed with $R e_{p}=0.001, \delta_{f} / \delta x=4$, and $\rho_{p} / \rho_{f}=1000$, and the errors are computed over a time period ranging from 0 to $100 \tau_{p}$.

\begin{tabular}{|l|l|l|l|l|}
\hline$\delta_{f} / d_{p}$ & 2 & 4 & 8 & 16 \\
\hline No corrections & 47.4 & 24.3 & 11.8 & 6.13 \\
\hline Volume fraction correction & 42.3 & 23.6 & 11.8 & 6.12 \\
\hline Fluid velocity correction & 6.89 & 2.09 & 0.591 & 0.537 \\
\hline Both corrections & 3.95 & 1.61 & 0.548 & 0.531 \\
\hline
\end{tabular}

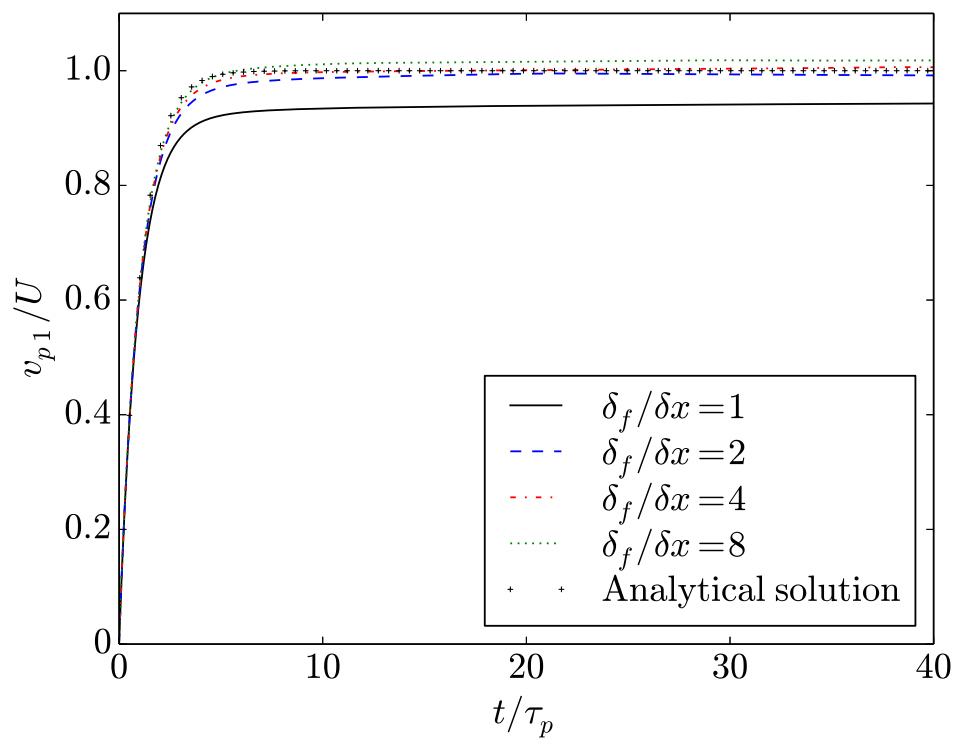

Figure 5: Particle settling speeds with the drag corrections from $\S 4$ for $\delta_{f} / d_{p}=8, R e_{p}=0.001, \rho_{p} / \rho_{f}=1000$, and various filter resolutions $\delta_{f} / \delta x$. The results are compared to the analytical (Stokes) solution.

\subsection{Effect of the filter resolution}

We next examine the effect of the filter resolution $\delta_{f} / \delta x$ on the residual drag corrections. Both the VFEL framework (§2.2) and our residual drag corrections $(\S 4)$ are derived under the assumption that our filter is adequately resolved on the computational grid (i.e., $\delta_{f} / \delta x \gg 1$ ). For a filter width $\delta_{f}=8 d_{p}$, we therefore test four different filter resolutions: $\delta_{f} / \delta x=1,2,4,8$. We expect that for sufficiently large $\delta_{f} / \delta x$, the results will be independent of the filter resolution.

From figure 5 and table 2, we observe accurate results for the particle settling velocity for $\delta_{f} / \delta x \geq 2$. We also find that the results for large $\delta_{f} / \delta x$ are mostly independent of the filter resolution, as expected. While we expect the errors to decrease uniformly with increasing $\delta_{f} / \delta x$, we do observe a slight increase in the errors for $\delta_{f} / \delta x=8$. These slight errors in this case are likely due to the details of our numerical implementation of the VFEL approach (e.g., the interpolation, time integration, forcing, and approximate filtering schemes usedsee $[32,23]$ for further information). 
Table 2: Percent root-mean-square error in $v_{p_{1}} / U$ for different values of $\delta_{f} / \delta x$. All simulations are performed using both corrections and with $R e_{p}=0.001, \delta_{f} / d_{p}=8$, and $\rho_{p} / \rho_{f}=1000$. The errors are computed over a time period ranging from 0 to $100 \tau_{p}$.

\begin{tabular}{|l|l|l|l|l|}
\hline$\delta_{f} / \delta x$ & 1 & 2 & 4 & 8 \\
\hline \% root-mean-square error & 5.80 & 0.940 & 0.548 & 1.78 \\
\hline
\end{tabular}

Table 3: Percent root-mean-square error in $v_{p_{1}} / U$ for different values of $\rho_{p} / \rho_{f}$. All simulations are performed using both corrections and with $R e_{p}=0.001, \delta_{f} / d_{p}=8$, and $\delta_{f} / \delta x=4$. The errors are computed over a time period ranging from 0 to $100 \tau_{p}$.

\begin{tabular}{|l|l|l|l|}
\hline$\rho_{p} / \rho_{f}$ & 250 & 1000 & 4000 \\
\hline$\%$ root-mean-square error & 0.821 & 0.548 & 0.616 \\
\hline
\end{tabular}

\subsection{Effect of the density ratio}

Next, we examine the effect of the ratio between the particle and fluid densities, $\rho_{p} / \rho_{f}$, on the proposed correction scheme. [26] showed that the particle dynamics are dependent on the ratio between the particle response time $\left(\tau_{p}\right)$ and the relaxation time of the grid $\left(\delta x^{2} / \nu_{f}\right)$, which they write as a grid-dependent Stokes number, $S t_{\delta}$. (Note that the grid-dependent Stokes number, $S t_{\delta}$, is distinct from the bodyforce Stokes number, $S t_{\text {body }}$, whose effect will be assessed in $§ 5.5$.) From $§ 5.1$ and $§ 5.2$, we saw that for the VFEL method and adequately resolved filters, the results are dependent on $\delta_{f}$ but generally not on $\delta x$. If we assume that the relaxation time of the grid, $\tau_{\text {visc }}$, is given as $\delta_{f}^{2} / \nu_{f}$, we can write

$$
S t_{\delta} \equiv \frac{\tau_{p}}{\tau_{\text {visc }}} \propto \frac{\rho_{p}}{\rho_{f}}\left(\frac{d_{p}}{\delta_{f}}\right)^{2} .
$$

Our velocity correction scheme in $\S 4.3$ assumes that the disturbance flow diffuses instantly, which can be thought of as $\tau_{\text {visc }} \rightarrow 0$, which is equivalent to $S t_{\delta} \rightarrow \infty$. We therefore expect that our residual drag correction will perform best when $\rho_{p} / \rho_{f}$ is large.

To assess the effectiveness of our residual drag correction at various density ratios, we therefore simulate cases with $\rho_{p} / \rho_{f}=250,1000$, and 4000. (Note that while we expect additional discrepancies for $\rho_{p} / \rho_{f}<250$, we have not simulated such cases, since we would then need to include additional history, added mass, and fluid acceleration terms in our particle equation of motion; see [7].) The results of our simulations are given in figure 6 and table 3. As in many of the previous cases, we have used $R e_{p}=0.001, \delta_{f} / d_{p}=8$, and $\delta_{f} / \delta x=4$.

At long times $\left(t / \tau_{p} \gtrsim 20\right)$, the results for all three density ratios approach the analytical predictions, and our time-averaged errors in table 3 show little difference with varying $\rho_{p} / \rho_{f}$. At early times $\left(t / \tau_{p} \lesssim 10\right)$, we notice slight errors in the predicted settling velocities. We see that error in the predicted settling velocity is largest for the smallest density ratio, as expected. In this case, the velocity disturbance diffuses less rapidly; the fluid velocity correction scheme, however, assumes that this disturbance has fully diffused, and therefore over-corrects the drag, causing the particle velocity to fall slightly below the analytical solution. 


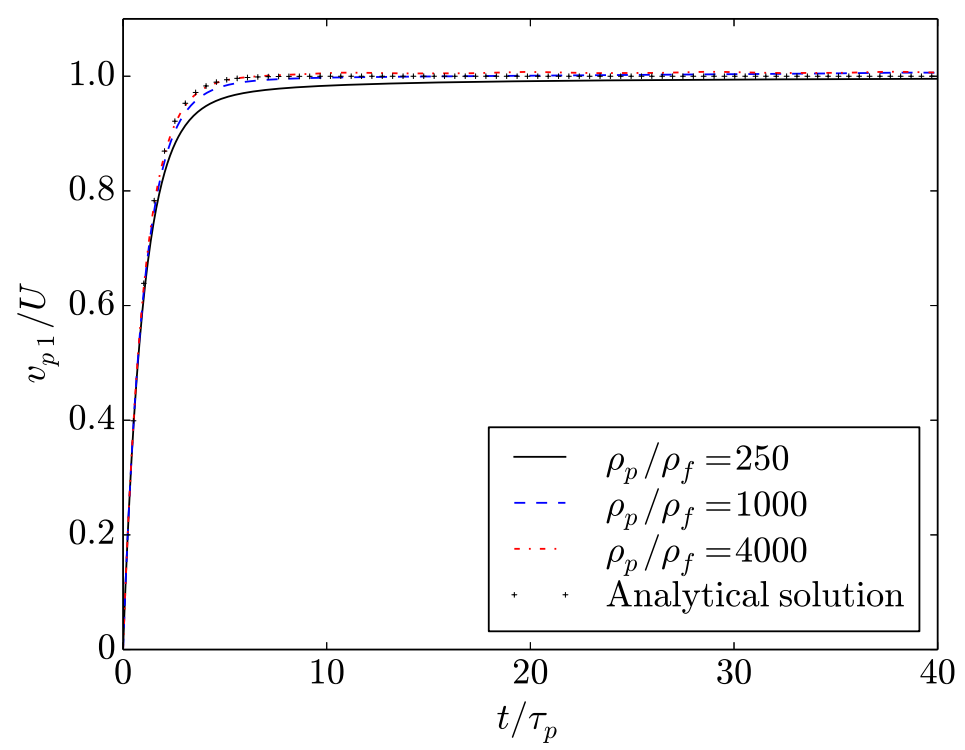

Figure 6: Particle settling speeds with the drag corrections from $\S 4$ for $\delta_{f} / d_{p}=8, R e_{p}=0.001, \delta_{f} / \delta x=4$, and various values of $\rho_{p} / \rho_{f}$. The results are compared to the analytical (Stokes) solution.

\subsection{Effect of the particle Reynolds number}

Our investigations up to this point have been limited to low-Reynolds-number flows; in particular, flows where $R e_{p}=0.001$. We now test our correction scheme at higher values of $R e_{p}$. Note that the fluid velocity corrections in $\S 4.2$ were derived under the assumption that the velocity disturbance due to the particle could be represented by the steady-state Stokes solution for the fluid velocity, given by (A1).

We thus increase $R e_{p}$ and study the effectiveness of our corrections as inertial forces become non-negligible. Note that for this study, as in [26], our approach is one of verification and not of validation. That is, we prescribe a drag law and then determine how accurately we are able to simulate the particle settling velocity this drag law should yield. (We are not concerned with the equally important question of whether the specified drag law is appropriate; this is a validation question that is beyond the scope of the present investigation but is addressed in several recent studies [34, 35, 14].) For the purposes of this study, our residual drag expression will assume Stokes drag; that is, (40) will always be calculated with $f_{D}=1$, regardless of the Reynolds number. We expect, however, that at large Reynolds numbers, a settling particle will induce non-negligible inertial forces through momentum coupling with the fluid. In this case, even when the drag on the particle is calculated using a Stokes drag law, the momentum coupling between the phases will likely result in a fluid velocity disturbance that does not match the Stokes velocity solution given by (A1). When this occurs, our corrected residual drag expression will likely lead to predictions for the particle settling velocity that do not match the analytical result. Here, we are interested in determining the range of Reynolds numbers over which we are able to recover particle settling velocities that match the analytical solution resulting from the prescribed Stokes drag law.

Since our simulations are performed with triply periodic boundary conditions, we must exercise care when simulating larger values of $R e_{p}$. In particular, we expect the wake behind 
Table 4: Percent root-mean-square error in $v_{p_{1}} / U$ for different values of $R e_{p}$. All simulations are performed with $\delta_{f} / d_{p}=8, \delta_{f} / \delta x=4$, and $\rho_{p} / \rho_{f}=1000$. The errors are computed over a time period ranging from 0 to $100 \tau_{p}$.

\begin{tabular}{|l|l|l|l|l|l|l|l|l|}
\hline$R e_{p}$ & 0.001 & 0.01 & 0.1 & 0.25 & 0.5 & 1 & 2 & 4 \\
\hline No corrections & 11.8 & 11.9 & 11.0 & 9.27 & 7.40 & 5.33 & 3.50 & 2.18 \\
\hline Both corrections & 0.548 & 0.644 & 0.535 & 2.03 & 3.84 & 5.90 & 7.80 & 9.22 \\
\hline
\end{tabular}

a particle to scale as $\tau_{p}^{2}|\boldsymbol{g}| \propto \tau_{p} R e_{p}[40,23]$. We therefore increase our domain size $\mathcal{L}$ as $R e_{p}$ increases such that $\mathcal{L} \gg \tau_{p}^{2}|\boldsymbol{g}|$. In the simulations below, we ensure that $\mathcal{L}>4.5 \tau_{p}^{2}|\boldsymbol{g}|$. We also set $\delta_{f} / d_{p}=8, \delta_{f} / \delta x=4$, and $\rho_{p} / \rho_{f}=1000$.

The results of our Reynolds-number study are shown in figure 7 and table 4 . We observe that for $R e_{p} \lesssim 0.1$, the normalized settling velocities are independent of $R e_{p}$, and that with the residual drag corrections, we are able to recover the analytical prediction almost exactly. For $R e_{p} \gtrsim 0.25$, the error in the uncorrected settling velocity predictions decreases with increasing $R e_{p}$. One possible explanation is that as $R e_{p}$ increases, inertial forces become significant and act to convect the induced fluid velocity disturbance away from the particle. Since the fluid velocity correction neglects convective effects, it over-predicts the local fluid velocity disturbance, and thus over-corrects for its effect on the drag. Indeed, from table 4 we notice that for $R e_{p} \gtrsim 1$, the error in the settling velocity prediction is larger when the correction is included than when it is omitted, highlighting the fact that our fluid velocity correction scheme is only suitable for low values of $R e_{p}$.

While this scheme could be empirically adjusted for higher values of $R e_{p}$ (e.g., by computing a Reynolds-number dependent fluid velocity correction), any such approach must be treated with caution. In particular, our fluid velocity corrections are formulated under the assumption the flow field can be represented as a linear superposition of Stokes velocity disturbances. Under that assumption, we can readily remove the effect of the disturbance in question and compute the undisturbed velocity. However, at larger $R e_{p}$, the induced velocity from a particle will interact nonlinearly with the flow and with other disturbance velocities. In that case, these interactions will depend on the locations of nearby particles and the precise flow patterns; while a correction scheme could be devised for a single particle in a uniform flow with large $R e_{p}$, it is unlikely that such an approach would generalize to arbitrary flow and particle configurations. For that reason, we have not attempted to formulate a correction scheme for intermediate and large $R e_{p}$. We simply recommend (based on figure 7 and table 4 ) that our correction scheme be used for $R e_{p} \lesssim 0.5$ and note that for $R e_{p} \gtrsim 4$, no correction scheme seems to be necessary.

\subsection{Effect of unsteadiness}

We finally consider the effect of $S t_{\text {body }}$ on the particle settling velocities. This will provide a simple test to allow us to estimate the effects on flow unsteadiness on the residual drag correction approach. Note that the previous tests were run with a constant bodyforce, which is equivalent to the limits $\tau_{\text {body }} \rightarrow \infty$ and $S t_{\text {body }} \rightarrow 0$. For finite $S t_{\text {body }}$, the analytical solution for the particle settling velocity is given in [26],

$$
\frac{v_{p_{1}}}{U}=\frac{S t_{\text {body }}}{1+S t_{\text {body }}^{2}}\left[\exp \left(-\frac{t}{\tau_{p}}\right)-\cos \left(\frac{t}{\tau_{\text {body }}}\right)\right]+\frac{1}{1+S t_{\text {body }}^{2}} \sin \left(\frac{t}{\tau_{\text {body }}}\right) .
$$



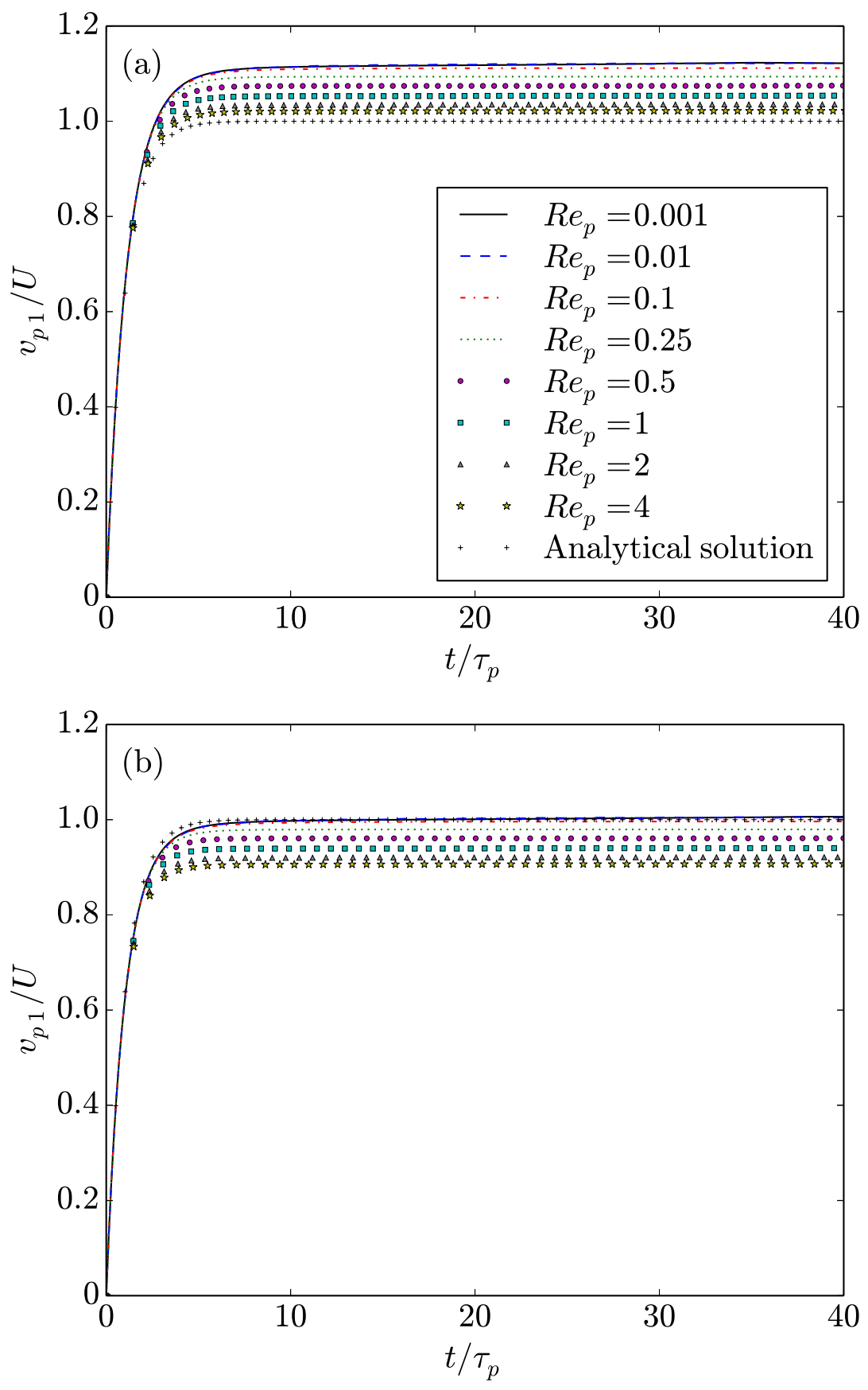

Figure 7: Particle settling speeds for various values of $R e_{p}$. The results in (a) are without any drag corrections, while the results in (b) are with both drag corrections from $\S 4$. All data have $\delta_{f} / d_{p}=8, \delta_{f} / \delta x=4$, and $\rho_{p} / \rho_{f}=1000$. The results are compared to the analytical (Stokes) solution. 
Table 5: Percent root-mean-square error in $v_{p_{1}} / U$ for different values of $S t_{\text {body }}$. All simulations are performed with $R e_{p}=0.001, \delta_{f} / d_{p}=8, \delta_{f} / \delta x=4$, and $\rho_{p} / \rho_{f}=1000$. The errors are computed over a time period ranging from 0 to $100 \tau_{p}$.

\begin{tabular}{|l|l|l|l|l|}
\hline$S t_{\text {body }}$ & 0 & 0.5 & 1 & 2 \\
\hline No corrections & 11.8 & 5.32 & 2.64 & 0.514 \\
\hline Both corrections & 0.548 & 1.58 & 1.54 & 1.19 \\
\hline
\end{tabular}

We consider three values for the bodyforce Stokes number: $S t_{\text {body }}=0.5,1,2$. In addition, we select $\delta_{f} / d_{p}=8, \delta_{f} / \delta x=4, \rho_{p} / \rho_{f}=1000$, and $R e_{p}=0.001$. (The particle Reynolds number $R e_{p}$ is defined here based on the peak amplitude of the bodyforce.)

Simulation results are shown in figure 8, and several inset plots are used to emphasize the settling velocities at particular times. The root-mean-square errors in $v_{p_{1}} / U$ are also tabulated in table 5 . We notice that for $S t_{\text {body }}=0.5$, the drag corrections improve the settling velocity predictions at all times (see figure 8(a)), leading to a lower root-meansquare error in the settling velocity (see table 5). At $S t_{\text {body }}=1$, the drag corrections improve the settling velocity predictions at early times (see the top inset plot in figure 8(b)). The results are also closer to the analytical solution near inflection points in the solution (see the middle inset plot in figure $8(\mathrm{~b})$ ). At long times, the settling velocities have similar errors both with and without the corrections near peaks in the solution (see the bottom inset plot in figure 8(b)). The overall result is a slightly lower value of the root-mean-square settling velocity error when the drag corrections are used (table 5 ). For $S t_{\text {body }}=2$, the settling velocity corrections tend give errors which are equal to or worse than those without the corrections at the different times shown (see figure 8(c)). The corrections thus lead to a slightly larger root-mean-square settling velocity error (table 5), though the errors remain small both with and without the corrections.

These results are expected, since our drag corrections are formulated under the assumption of steady-state conditions. When the particle response time is small compared to the timescale of the bodyforce $\left(S t_{\text {body }} \lesssim 1\right)$, this assumption remains appropriate, and the drag corrections produce reasonable results. However, when the bodyforce varies more rapidly than the particle response time $\left(S t_{\text {body }} \gtrsim 1\right)$, our assumption of steady-state conditions is not reasonable, we over-predict the local fluid velocity disturbance from the particle, and we thus slightly over-correct the drag.

We thus observe that our drag corrections are appropriate, provided that the unsteadiness of the system is characterized by a timescale comparable to or greater than the particle response time. (If this condition is not met, our drag corrections lead to worse predictions in the particle settling velocity, though the errors still remain small.) For a realistic turbulent flow, we hypothesize that this condition should hold in of possible scenarios. First, if $\tau_{p} \lesssim \tau_{f}$ (where $\tau_{f}$ is the characteristic time of large eddies driving particle motion in the flow), then changes in the flow will occur on timescales above the particle response time, and thus our assumption of steady-state conditions is appropriate. Note that the appropriate eddy size contributing to particle motion will be flow-dependent. In flows where particle motion is driven by Kolmogorov-scale centrifuging mechanisms (e.g., see [41]), we expect $\tau_{f} \sim \tau_{\eta}$, where $\tau_{\eta}$ is the Kolmogorov timescale. In CIT flows that are dominated by clusterscale momentum exchange between the phases $[22,23]$, however, we expect the $\tau_{f} \sim T_{L}$, 

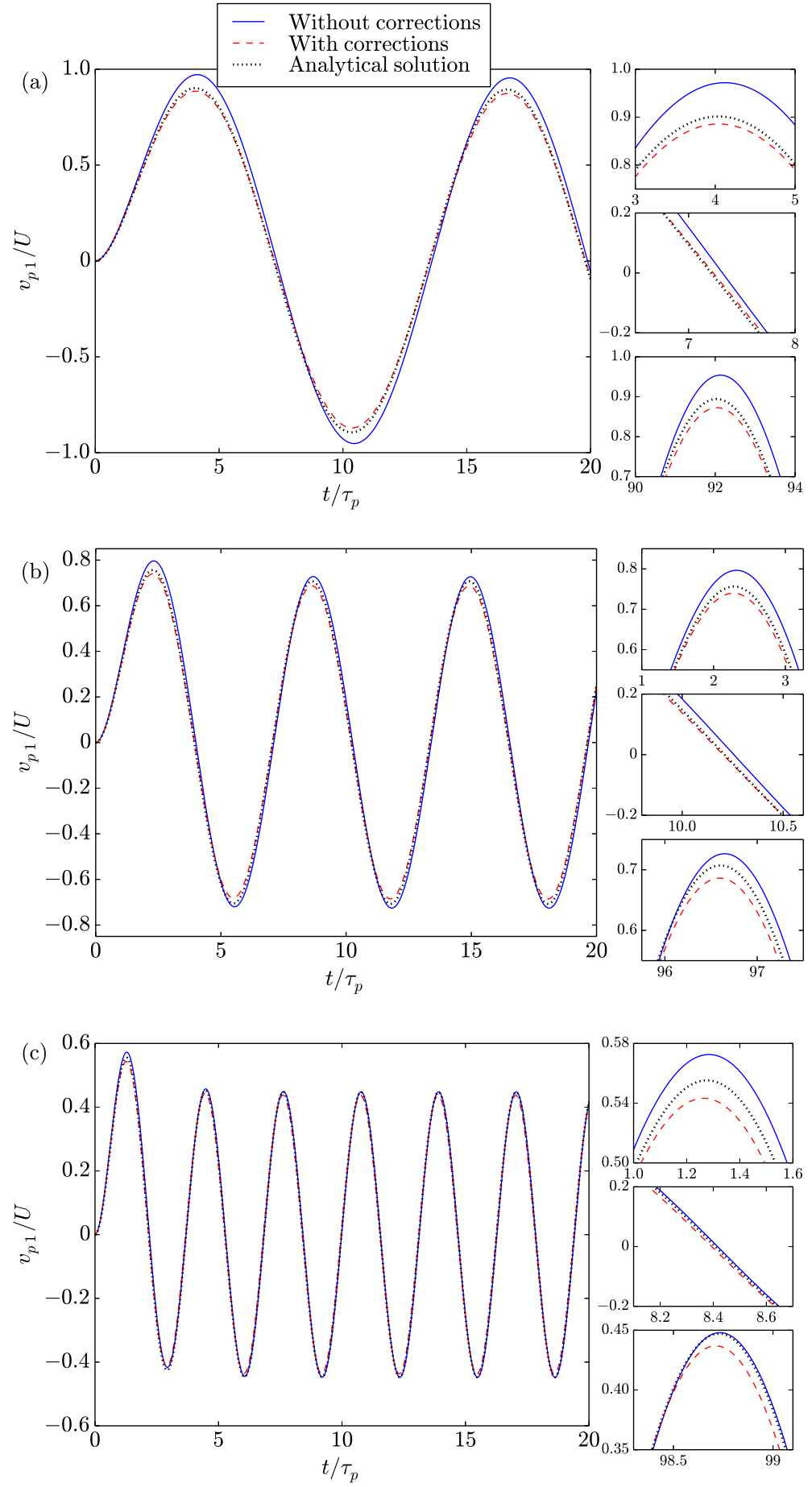

Figure 8: Particle settling speeds with and without the drag corrections for $\delta_{f} / d_{p}=8, R e_{p}=0.001$, $\delta_{f} / \delta x=4$, and various values of $S t_{\text {body }}$. (a) shows $S t_{\text {body }}=0.5$; (b) shows $S t_{\text {body }}=1$; (c) shows $S t_{\text {body }}=2$. The results are compared to the analytical (Stokes) solution. The insets show comparisons at particular times. 
where $T_{L}$ is the large-eddy timescale.

Second, if $\tau_{p}|\boldsymbol{g}| \gtrsim \mathcal{U}_{f}$ (where $\mathcal{U}_{f}$ is characteristic velocity of large eddies in the flow), then the particle velocities are primarily determined by the constant gravitational force, and are less affected by unsteady, turbulent motions. If either or both of these two conditions hold, then the residual drag corrections are expected to yield improved settling velocity predictions.

\subsection{Drag correction applicability for environmental and industrial flows}

In concluding this section, it is helpful to review the range of applicability of the drag corrections and then to discuss environmental and industrial flows for which they are expected to be relevant. The relevant parameters for which the corrections are appropriate are enumerated below.

1. Geometric and boundary conditions

Our drag corrections are formulated for spherical particles in unbounded domains.

2. Filter size and resolution

The drag corrections were found to be improve settling velocity predictions for filter sizes $\delta_{f} / d_{p} \geq 2$ and for filter resolutions $\delta_{f} / \delta x \geq 2$.

3. Particle and fluid densities

For $\rho_{p} / \rho_{f} \geq 250$, the corrections improved settling velocity predictions. (Note that smaller density ratio regimes were not tested, since the particle equation of motion used to formulate the drag corrections is not applicable for these regimes.)

4. Particle Reynolds number

Our drag corrections were found to improve the settling velocity predictions for particle Reynolds numbers where Stokes drag is accurate, that is, for $R e_{p} \lesssim 0.5$ [42]. Outside of this regime, the drag on a particle varies nonlinearly based on the turbulent flow field and on the positions of other particles, and thus no simple correction is expected to be appropriate.

5. Particle Stokes number

We expect that our drag corrections will lead to improve settling velocity predictions if the Stokes number $S t \equiv \tau_{p} / \tau_{f} \lesssim 1$. $\left(\tau_{f}\right.$ is the timescale of the eddies primarily responsible for particle motion, and depending on the flow considered, can range from the Kolmogorov timescale to the large-eddy timescale.) Alternatively, if the above condition is not met, the corrections may also allow for accurate settling velocity predictions when $\tau_{p}|\boldsymbol{g}|$ is greater than or about equal to the characteristic velocity of large eddies in the flow.

While the conditions above may seem restrictive, they are in fact present in several environmental and industrial flows. We now consider two active research topics - droplet growth in warm, cumulus clouds and particle motion in industrial risers - to assess the range of applicability of the correction approach in realistic flow scenarios.

One longstanding question in the cloud physics community is why droplets in warm, cumulus clouds grow more quickly than predicted by existing microphysical models [43, 44, 45]. In particular, researchers are unsure of the mechanisms by which droplets grow larger

than $30 \mu \mathrm{m}$, with the common hypothesis being that droplet growth in this range is due to 
turbulent processes [45]. Above $80 \mu \mathrm{m}$, droplet growth is expected to be due to predictable gravity-induced collisions.

We therefore test the applicability of our drag corrections in this regime based on the conditions above. For water droplets in air, we have $\rho_{p} / \rho_{f} \sim 1000, \mu_{f} \sim 0.015 \mathrm{~kg} /(\mathrm{m} \cdot \mathrm{s})$, and $\epsilon \sim 0.01 \mathrm{~m}^{2} / \mathrm{s}^{3}$ (see [43]). For $30 \mu \mathrm{m}$ droplets, we therefore find that $R e_{p} \sim 0.07$ and $S t \sim 0.09$ (with $S t$ being defined here based on $\tau_{\eta}$ ). We find that droplets with diameters of $60 \mu \mathrm{m}$ are characterized by $R e_{p} \sim 0.5$ and $S t \sim 0.3$; for larger droplet diameters, our drag corrections are therefore no longer appropriate. Our drag corrections are thus useful in providing more accurate simulations of droplets with diameters less than $60 \mu \mathrm{m}$, which includes much of the range that is currently problematic to cloud physics researchers.

Next, we consider the case of industrial riser flows. We use the study of [46] to obtain parameter estimates for these flows. Taking $\rho_{p} / \rho_{f} \sim 1500$ and $\mu_{f} \sim 0.015 \mathrm{~kg} /(\mathrm{m} \cdot \mathrm{s})$, we find that $R e_{p} \lesssim 0.5$ for $d_{p} \lesssim 50 \mu \mathrm{m}$, indicating that the drag correction is not appropriate for particles with diameters larger than $50 \mu \mathrm{m}$. We now test if this limit must be reduced based on the requirement that $S t \lesssim 1$.

We expect that for industrial risers, much like in CIT (see $\S 5.5$ and $\S 6$ ), particle motion is governed by large-scale turbulent eddies with timescale $T_{L}$. While no estimate for $T_{L}$ is provided in [46], we know that the turbulent Reynolds number for this flow is $10^{4}-10^{6}$ and that $\tau_{\eta} \sim 0.01 \mathrm{~s}$. We therefore can confidently conclude that $T_{L}$ is well above $0.015 \mathrm{~s}$ (the particle response time for $50 \mu \mathrm{m}$ particles), and thus that $S t \ll 1$ whenever $R e_{p} \leq 0.5$.

In a typical riser experiment (e.g., see [47]), about $10 \%$ of the overall particles have diameters below $50 \mu \mathrm{m}$. Therefore, while the correction approach described here may not be applicable for most of the particle sizes in a typical riser flow, it is expected to lead to improved predictions of interphase coupling and particle settling velocities when applied selectively on particles with $d_{p} \lesssim 50 \mu \mathrm{m}$.

\section{Cluster-induced turbulence}

Having verified our residual drag corrections for some simple flow scenarios, we now study their effect on the more complicated test case of cluster-induced turbulence (CIT). Our CIT simulations are performed in three dimensions with periodic boundary conditions on all sides. Particles at rest are placed randomly in an initially quiescent flow field. As the simulation progresses, gravity accelerates the particles, causing them to exchange momentum with the underlying flow field. This in turn leads to turbulent-like fluid velocity fluctuations and largescale particle clustering, as discussed in $[22,23]$. The parameters for our CIT simulations are given in table 6 . (Note that we use $\alpha_{p}$ to denote the particle volume fraction: $\alpha_{p} \equiv 1-\alpha_{f}$.) These parameters were selected so as to remain within the range over which our methodology is appropriate (see $\S 5)$. As in $[22,23]$, a soft-sphere model with a restitution coefficient $e=0.9$ is used to simulate particle collisions, and a mean pressure gradient is added to the momentum equation to force the mean fluid velocities to be zero in all directions. The simulations were performed using a Stokes drag law; i.e, $f_{D} \equiv 1$ in (40).

We perform two simulations: one without the fluid volume fraction and fluid velocity corrections, and one with both the fluid velocity and fluid volume fraction corrections. The simulations were run until the fluid and particle statistics reached a statistically stationary 
Table 6: Parameters for the CIT simulations. $\mathscr{L}$ denotes the cluster lengthscale, $\tau_{p}^{2}|\boldsymbol{g}|$ (see $\S 5.4$ ).

\begin{tabular}{|l|l|l|}
\hline Name & Notation & Value \\
\hline Filter size & $\delta_{f} / d_{p}$ & 8 \\
\hline Filter resolution & $\delta_{f} / \delta x$ & 4 \\
\hline Density ratio & $\rho_{p} / \rho_{f}$ & 1000 \\
\hline Particle Reynolds number & $R e_{p}$ & 0.1 \\
\hline Mean particle volume fraction & $\left\langle\alpha_{p}\right\rangle$ & 0.01 \\
\hline Normalized domain length (in $x$ ) & $\mathcal{L}_{x} / \mathscr{L}$ & 184 \\
\hline Normalized domain length (in $y$ ) & $\mathcal{L}_{y} / \mathscr{L}$ & 46.1 \\
\hline Normalized domain length (in $z)$ & $\mathcal{L}_{z} / \mathscr{L}$ & 46.1 \\
\hline Grid points in $x$-direction & $N_{x}$ & 512 \\
\hline Grid points in $y$-direction & $N_{y}$ & 128 \\
\hline Grid points in $z$-direction & $N_{z}$ & 128 \\
\hline
\end{tabular}

Table 7: Particle mean settling velocities from the CIT simulation, both with and without the fluid volume fraction and fluid velocity corrections. The statistics are computed over times ranging from $160 \tau_{p}$ to $260 \tau_{p}$. We have included error bars representing $95 \%$ confidence intervals about the sample means.

\begin{tabular}{|l|l|l|}
\hline Quantity & Without corrections & With corrections \\
\hline$\left\langle v_{p_{1}}\right\rangle^{*} / U$ & $2.68 \pm 0.0117$ & $2.53 \pm 0.00342$ \\
\hline
\end{tabular}

state. After statistical stationarity had been obtained (taken to be for times greater than $\left.160 \tau_{p}\right)$, the simulations were continued for an additional $100 \tau_{p}$.

In figure 9, we show instantaneous fluid velocity and particle volume fraction fields at a time of $200 \tau_{p}$. We compare the results when no corrections are used (left image) to the result when both corrections are used (right image). As noted in [23], regions of downward velocity (indicated by positive $u_{f_{1}} / U$, to remain consistent with our earlier definitions) tend to correlate with regions of high particle concentration. Apart from statistical fluctuations in the results, however, it is difficult to discern any significant difference between the two cases.

To better understand the effect of the drag corrections on the system properties, we first plot the mean particle settling velocity,

$$
\left\langle v_{p_{1}}(t)\right\rangle^{*} \equiv \frac{1}{N_{p}} \sum_{n=1}^{N_{p}} v_{p 1}^{(n)}(t),
$$

as a function of time in figure 10. From figure 10, we notice that the corrections tend to decrease the particle settling velocities, as expected. In table 7, we report time-averaged settling velocities once the simulations have reached a statistically stationary state. (Statistical averages are computed over times ranging from $160 \tau_{p}$ to $260 \tau_{p}$ ). From table 7 , we see that the drag corrections lead to a statistically significant decrease in the mean particle settling velocities.

To understand the effect of the corrections on other system statistics, we also computed time-averages of the particle fluctuation energy, the particle granular temperature, the fluid kinetic energy, and the partial radial distribution functions. None of these quantities, how- 

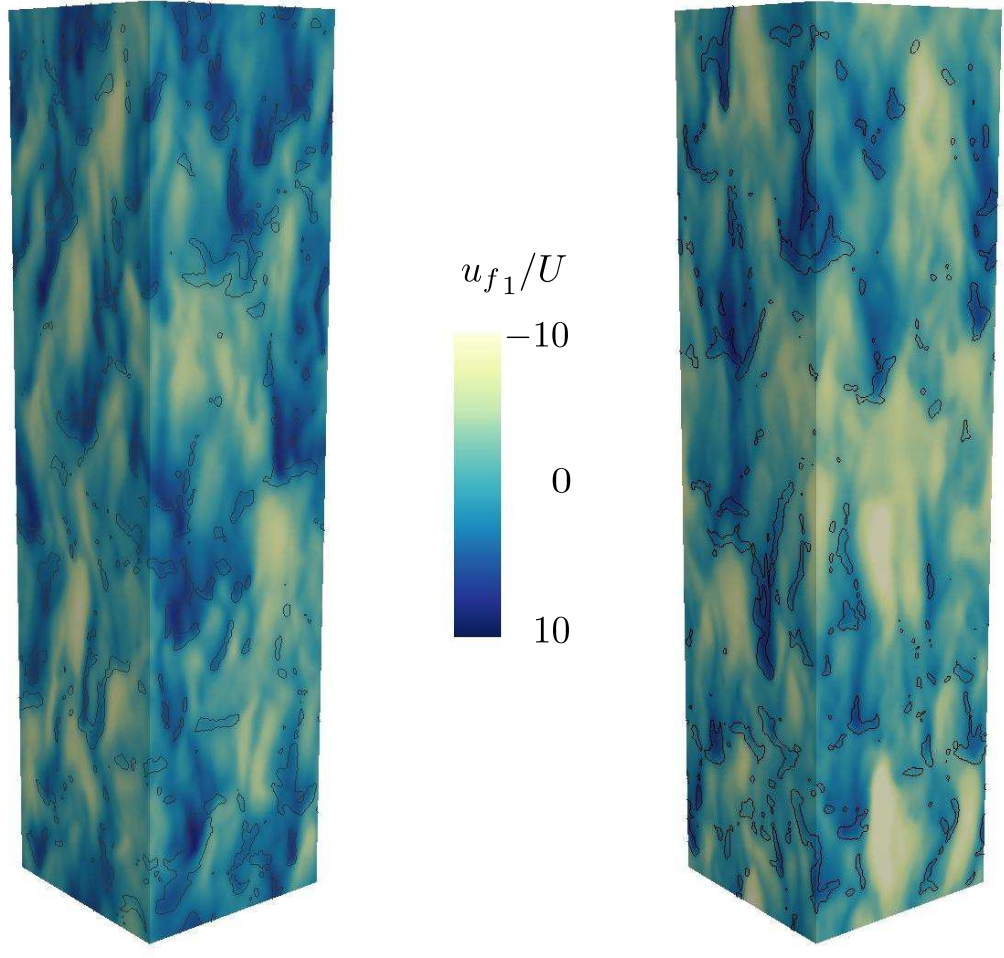

Figure 9: Instantaneous snapshots of the fluid velocity in the direction of gravity and the particle volume fraction. Gravity is oriented in the downward direction. The fluid velocity (taken to be positive in the downward direction) is indicated by the color contours, and regions of large particle volume fraction (twice the mean value) are denoted by the black contour lines. Data are shown without the fluid velocity and fluid volume fraction corrections (left) and with the fluid velocity and fluid volume fraction corrections (right) at a time of $200 \tau_{p}$. 


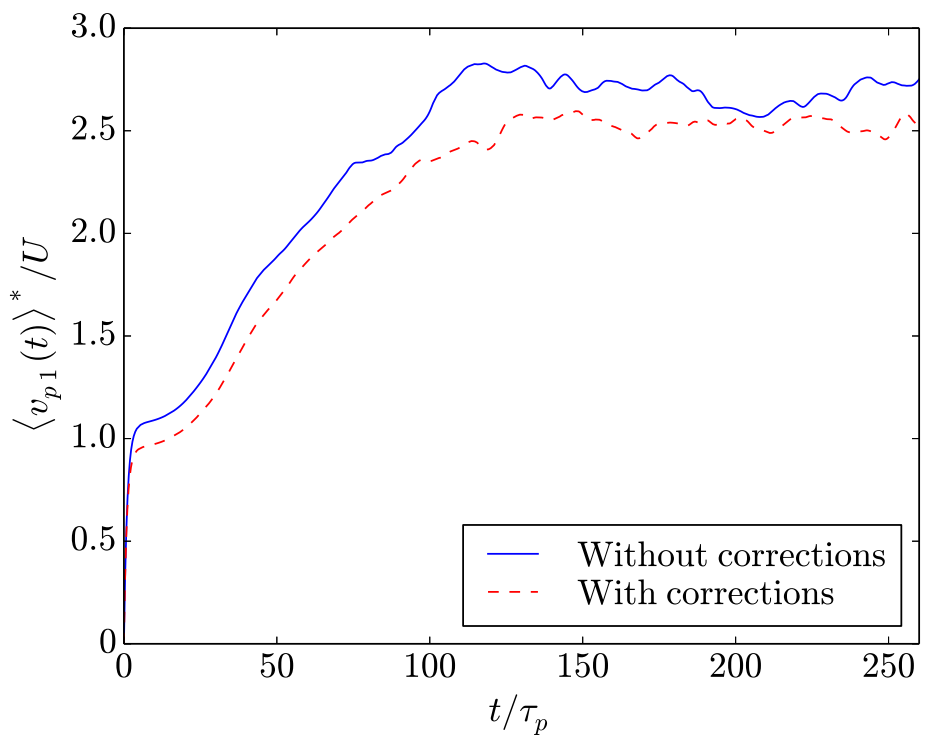

Figure 10: The average particle settling velocity $\left\langle v_{p_{1}}(t)\right\rangle^{*}$, plotted as a function of time for the CIT simulations both with and without the drag corrections.

ever, showed a statistically significant difference when the drag corrections were introduced, and therefore they are not reported here.

We now verify that the Stokes and Reynolds numbers are appropriate for the correction scheme to be applied. The particle Reynolds number $R e_{p}$ (based on the average particle setting velocity) is 0.27 , which is safely below the suggested upper limit of 0.5 . We next check the particle Stokes number. As discussed in [23], in both CIT and dilute particle-laden flows, particles accumulate outside of high vorticity regions. However, in CIT, these high vorticity regions are generated by shear layers between clusters, and not by the Kolmogorovscale centrifuging mechanism identified in [41]. We therefore expect the appropriate Stokes number characterizing particle motion in CIT to be the ratio between the particle response time $\tau_{p}$ and large-eddy (i.e., cluster-scale) timescale $T_{L}$, as noted in $\S 5.5$. We compute $T_{L}$ as the longitudinal integral lengthscale of the flow divided by the root-mean-square fluid velocity fluctuation. From our simulation results, we have $T_{L} \sim 0.2 \mathrm{~s}$, which gives us $S t=\tau_{p} / T_{L} \sim 0.1$.

Our simulations are therefore indeed within the appropriate parameter ranges for the correction to be safely applied. We thus expect that for this CIT configuration, the drag correction leads to improved particle settling velocity predictions.

\section{Conclusions}

In this study, we have introduced a simple, analytical approach for improving particle drag predictions for two-way and four-way coupled particle-fluid systems. In $\S 2$, we compared the VFEL method to traditional E-L methods. The VFEL method, unlike traditional E-L schemes, provides grid-independent predictions of the particle drag force at arbitrary particle loadings. We therefore briefly discussed some simple changes that could be made to recast traditional E-L schemes within the VFEL framework. In $\S 3$, we showed that the VFEL 
approach, like traditional E-L methods, does not provide accurate predictions of particle settling in the low Reynolds number limit. These errors result because the original formulation of the VFEL method uses filtered fluid velocity and volume fractions in the residual drag calculations, while instead it should be using undisturbed fluid velocity and volume fractions. In $\S 4$, we introduced analytical fluid volume fraction and fluid velocity corrections for three-dimensional, unbounded domains. These corrections are used to recover undisturbed quantities from filtered quantities. We tested the effectiveness of these corrections in $\S 5$, and we argued that they provide improved settling velocity predictions for $R e_{p} \lesssim 0.5, \delta_{f} / d_{p} \gtrsim 4$, $\delta_{f} / \delta x \gtrsim 2$, high density ratios, and for flows where $\tau_{p} \lesssim \tau_{f}$ or $\tau_{p}|\boldsymbol{g}| \gtrsim \mathcal{U}_{f}$. Finally, we ran two simulations of cluster-induced turbulence in $\S 6$, one without any corrections to the drag expression, and one where the fluid velocities and fluid volume fractions were adjusted based on the formulations in $\S 4$. We observed that the corrections led to a statistically significant decrease in the particle settling velocity, but had a negligible effect on the other fluid and particle statistics considered.

This study, by building on the work of [32], provides the first accurate and grid-independent Euler-Lagrange method for computing particle drag forces in low-Reynolds-number, two-way and four-way coupled flows. For a prescribed drag law, we have introduced and verified a drag correction scheme to allow the VFEL approach to recover the expected particle settling velocity for canonical flow scenarios. Our analytical drag correction approach can be easily introduced into a VFEL code, and only a few simple modifications are necessary to transform a traditional E-L code into a VFEL code. We hope that this improved numerical method will enable improvements in the design and understanding of engineering and environmental particle-fluid systems.

\section{Acknowledgements}

The authors are grateful to J. A. K. Horwitz, J. Capecelatro, R. G. Patel, R. O. Fox, and C. A. Whealton for many helpful discussions. This work was supported by the U.S. National Science Foundation under grant CBET-1437903. We would also like to acknowledge high-performance computing support from Yellowstone (ark:/85065/d7wd3xhc) provided by NCAR's Computational and Information Systems Laboratory, sponsored by the National Science Foundation [48].

\section{Appendix A. Resolved drag for various filter sizes and flow configurations}

In this section, we study the effect of the filter size $\delta_{f}$ on the resolved $\operatorname{drag} \boldsymbol{F}_{\text {resolved }}^{(n)}$. In Appendix A.1, we consider uniform Stokes flow around a sphere in the absence of a mean pressure gradient. We then add a mean pressure gradient to the system in Appendix A.2 to study how the VFEL framework handles buoyancy effects. Finally, in Appendix A.3, we consider a simple flow with two particles to assess our ability to resolve drag modifications resulting from the presence of additional particles.

\section{Appendix A.1. Stokes flow around a particle}

We first consider the simplest possible flow configuration: a Stokes flow around a single particle in the absence of a mean pressure gradient. We use this configuration because the 
analytical solution is known and the filtered velocity and pressure fields can be assessed independently of any errors in the numerical solution of the unfiltered fields. We take the origin of our coordinate system to coincide with the center of the particle, and we define the free stream velocity $\boldsymbol{U} \equiv(-U, 0,0)^{\top}$. The fluid velocity $\boldsymbol{u}_{\boldsymbol{f}} \equiv\left(u_{f_{1}}, u_{f_{2}}, u_{f_{3}}\right)^{\top}$, where the Cartesian velocity components $u_{f_{1}}, u_{f_{2}}$, and $u_{f_{3}}$ are given by

$$
\begin{aligned}
\frac{u_{f_{1}}}{U} & =-1+\frac{3}{4}\left(\frac{r_{p}}{r}\right)\left(1+\cos ^{2} \theta\right)+\frac{1}{4}\left(\frac{r_{p}}{r}\right)^{3}\left(1-3 \cos ^{2} \theta\right), \\
\frac{u_{f_{2}}}{U} & =\frac{3}{4}\left(\frac{r_{p}}{r}\right) \cos \theta \sin \theta \cos \phi\left[1-\left(\frac{r_{p}}{r}\right)^{2}\right], \\
\frac{u_{f_{3}}}{U} & =\frac{3}{4}\left(\frac{r_{p}}{r}\right) \cos \theta \sin \theta \sin \phi\left[1-\left(\frac{r_{p}}{r}\right)^{2}\right] .
\end{aligned}
$$

$r_{p}$ is the radius of the particle, $r$ is the radial distance from the center of the particle, $\phi$ is the azimuthal angle, and $\theta$ is the polar angle. The polar axis is taken to coincide and align with the positive $x_{1}$-axis. The Stokes solution to the pressure is given as

$$
\frac{p}{\mu_{f} U / r_{p}}=\frac{3}{2}\left(\frac{r_{p}}{r}\right)^{2} \cos \theta .
$$

To simplify our expressions (and without loss of generality), we have taken our free-stream pressure to be zero.

We now seek to compute the resolved forces $\boldsymbol{F}_{\text {resolved }}^{(n)}$. We do so by solving both (20) (which is valid for all filter sizes) and (21) (which is valid for $\delta_{f} \gg d_{p}$ ). These two expressions should be equivalent for $\delta_{f} \gg d_{p}$. To compute (20) and (21), we must filter the analytical predictions for the velocities and pressure, given by (A1) and (A2). The filtering is based on a numerical calculation of (11) using the adaptive quadrature routines provided in the SciPy package. To evaluate (20), we sample the filtered velocities and pressures discretely along the surface of the particle using a cylindrical mesh with 5760 points in the $\theta$-direction (with a uniform grid spacing of $0.03125^{\circ}$ in this direction) and two grid points in the $r$-direction (one on the surface of the sphere, one a distance $10^{-5} r_{p}$ outside of the sphere). This grid resolution is chosen to ensure that the filtered stresses do not change as the grid is refined. (Because of the axisymmetric nature of the problem, we do not need to sample the filtered quantities at different $\phi$-locations.) We evaluate (21) by differentiating the filtered velocities and pressures at the center of the particle. These quantities are sampled on a uniform grid with a spacing of $10^{-5} r_{p}$.

Note that while this numerical implementation of the filtering approach differs from the approximate implicit filtering implementation used in VFEL simulations [32], it is expected to be more accurate and to provide a more rigorous a priori testing of the framework described in $\S 2.2$.

We consider the ratio between the resolved drag force $\boldsymbol{F}_{\text {resolved }}$ and the total drag force $\boldsymbol{F}=\boldsymbol{F}_{\text {resolved }}+\boldsymbol{F}_{\text {residual }}$. (Note that we have dropped the superscript $(n)$, since we are only considering a system with a single particle.) The total drag force $\boldsymbol{F}$ can be broken into viscous stress and pressure contributions,

$$
\boldsymbol{F}=\boldsymbol{F}^{\text {viscous }}+\boldsymbol{F}^{\text {pressure }}
$$



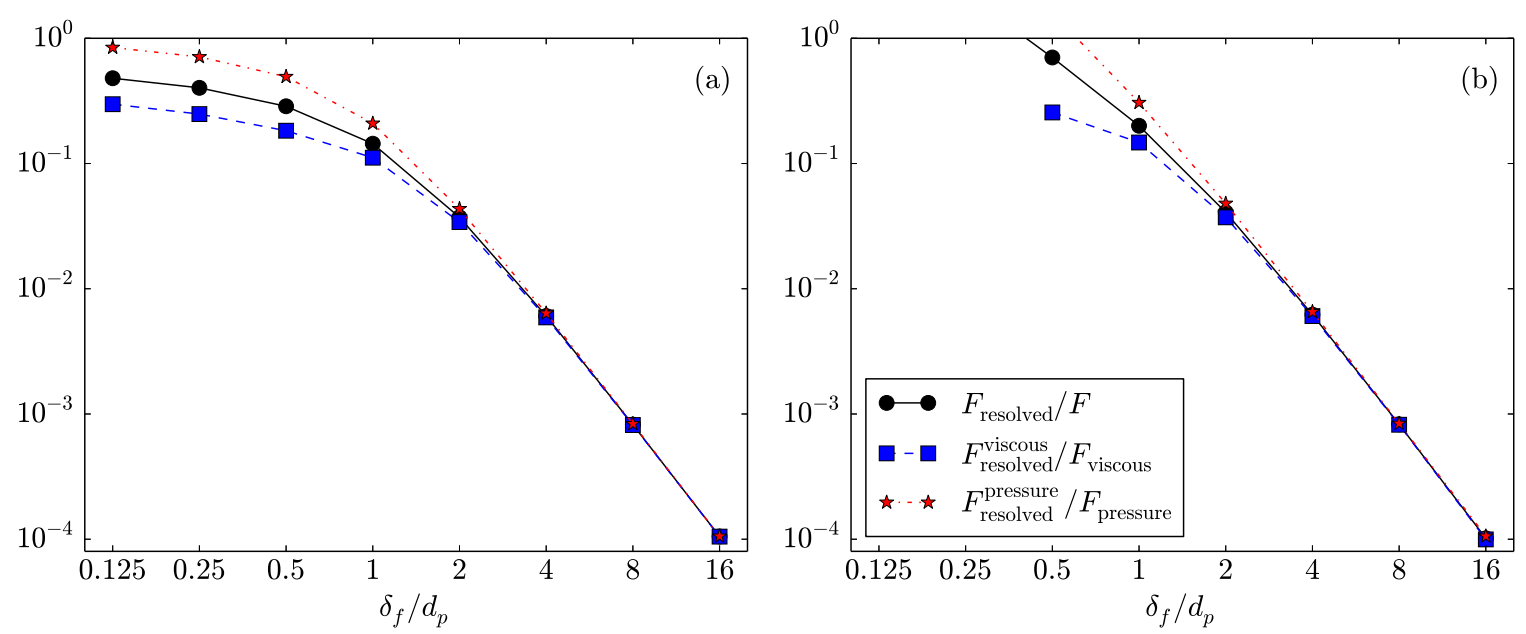

Figure A.11: The ratio of resolved to total drag for a uniform Stokes flow past a sphere for various filter sizes $\delta_{f}$. Also shown are the ratios between the resolved and total pressure drag contributions, and the resolved and total viscous drag contributions. The results in (a) are computed by numerically evaluating (20), while the results in (b) are computing using the approximate relation (21), which is expected to be valid in the limit of $\delta_{f} \gg d_{p}$.

each of which has a resolved and a residual component.

In figure A.11, we plot the ratio between the resolved drag and the total drag, the resolved viscous drag and the total viscous drag, and the resolved pressure drag and the total pressure drag for various filter sizes $\delta_{f}$. In figure A.11(a), these quantities are computed using (20) (the exact expression for the resolved drag), while in figure A.11(b), they are computed using (21) (the approximation for the resolved drag used in [32]). (Note that since the overall forces only act along the $x_{1}$-direction, we have dropped the bold-faced notation and use the regular type to denote the projection of the force along the $x_{1}$-direction.) Figure A.11 indicates that (20) and (21) give very similar results for $\delta_{f} / d_{p} \gtrsim 2$, and thus that (20) can be accurately approximated using (21) for large $\delta_{f} / d_{p}$. For $\delta_{f} / d_{p} \lesssim 2$, a non-negligible fraction of the overall drag is resolved, indicating that the model for the unresolved drag would need to vary based on $F_{\text {resolved }}$. However, it is important to note that our momentum conservation equation (14) was derived under the assumption that $\delta_{f} \gg d_{p}$, and is thus not valid in this regime. For typical VFEL runs (e.g., see $[22,23]$ ), however, $\delta_{f} / d_{p} \approx 8$. At this point, less than $0.1 \%$ of the overall drag is resolved, and thus we can safely formulate our residual drag expressions without needing to adjust for the resolved drag.

In closing this section, we note that the analysis above was conducted for Stokes flows, and is therefore only strictly valid in the limit where $R e_{p} \rightarrow 0$. However, we can use the predictions from [49] to extend this analysis to small $R e_{p}$. Based on [49], we can write

$$
\boldsymbol{F}_{\text {resolved }}\left(R e_{p}\right)=\left[1+\frac{3}{16} R e_{p}+\frac{9}{160} R e_{p}^{2} \ln \left(R e_{p} / 2\right)+\mathcal{O}\left(R e_{p}^{2}\right)\right] \boldsymbol{F}_{\text {resolved }}\left(R e_{p} \rightarrow 0\right) .
$$

We can therefore extend the results presented beyond the Stokes limit, allowing us to argue that we can safely use a standard microscale drag model at small but finite values of $R e_{p}$. 


\section{Appendix A.2. Stokes flow with a uniform pressure gradient}

We consider an extension of the analysis in Appendix A.1, where we now have superimposed a uniform, gravity-induced pressure gradient to the Stokes solution around a single particle. One motivation for looking at this type of flow is that the particle equation of motion for the VFEL simulations, (26), does not explicitly contain any terms to account for buoyancy effects, under the assumption is that buoyancy is implicitly included through the action of the resolved pressure gradients.

In our tests, the strength of the buoyant forces is specified through a non-dimensional Froude number, here defined as

$$
F r \equiv \frac{\mu_{f} U}{\rho g r_{p}^{2}}
$$

A lower value of $F r$ indicates a stronger pressure gradient and thus stronger buoyant forces.

The analytical velocity distribution is identical that specified earlier in (A1), while the pressure distribution is given as

$$
\frac{p}{\mu_{f} U / r_{p}}=\underbrace{\frac{3}{2}\left(\frac{r_{p}}{r}\right)^{2} \cos \theta}_{p_{\text {fluctuating }}}+\underbrace{\frac{1}{F r}\left(\frac{r}{r_{p}}\right) \cos \theta}_{p_{\text {mean }}} .
$$

The first term ( $\left.p_{\text {fluctuating }}\right)$ is the contribution from pressure fluctuations, while the second term $\left(p_{\text {mean }}\right)$ is the contribution from the mean pressure gradient.

In Appendix A.1, we measured the fraction of the fluctuating pressure drag that was resolved for various filter sizes. Now, we will measure the fraction of the mean pressure drag that is resolved. To do so, we define

$$
\boldsymbol{F}_{\text {mean }}^{\text {pressure }}=\int_{\mathcal{S}}-\boldsymbol{n} p_{\text {mean }} d \boldsymbol{y}
$$

and

$$
\boldsymbol{F}_{\text {resolved,mean }}^{\text {pressure }}=\int_{\mathcal{S}}-\boldsymbol{n} \overline{p_{\text {mean }}} d \boldsymbol{y}
$$

where $\boldsymbol{F}_{\text {mean }}^{\text {pressure }}$ is the total drag due to the mean pressure gradient, and $\boldsymbol{F}_{\text {resolved,mean }}^{\text {pressure }}$ is the resolved drag due to the mean pressure gradient. As in Appendix A.1, we can simplify (A8) by assuming $\delta_{f} \gg d_{p}$, which gives us

$$
\boldsymbol{F}_{\text {resolved,mean }}^{\text {presure }} \approx-\mathcal{V}_{p}^{(n)} \nabla \overline{p_{\text {mean }}} .
$$

Note that [32] calculate the resolved pressure gradients using (A9). We therefore seek to understand how accurate this approximation is for various filter sizes.

With the same numerical methods described in Appendix A.1, we use both (A8) and (A9) to compute the ratio $F_{\text {resolved,mean }}^{\text {pressure }} / F_{\text {mean }}^{\text {pressure }}$ for various filter sizes $\delta_{f}$ and plot the results (which are independent of $F r$ ) in figure A.12. (Note that as before, we have dropped the bold-faced notation, since we are considering only the force in the direction of the mean flow. The net force in the other directions is identically zero.)

(A8) and (A9) give similar predictions when $\delta_{f} / d_{p} \gtrsim 2$, indicating that (A9) is an appropriate approximation for large filter sizes. We also notice that the buoyant forces are slightly 


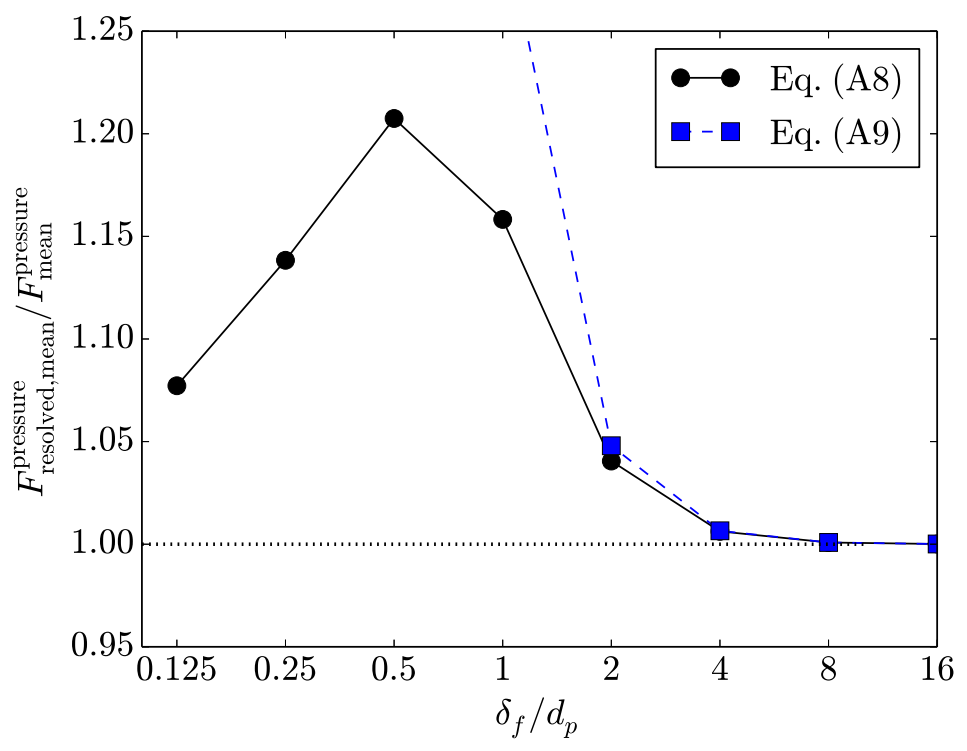

Figure A.12: The ratio of resolved to total drag from the mean pressure gradient for a uniform Stokes flow past a sphere for various filter sizes $\delta_{f}$. The results are computed using both (A8) (which is expected to be valid for all $\delta_{f}$ ) and (A9) (which is expected to be valid in the limit of $\delta_{f} \gg d_{p}$ ).

over-predicted at low values of $\delta_{f} / d_{p}$. However, the governing equations in the VFEL approach are derived for $\delta_{f} / d_{p} \gg 1$, and thus the VFEL simulations are typically performed at larger values of $\delta_{f} / d_{p}$. For $\delta_{f} / d_{p}=8$ (a typical value in VFEL simulations), we see that $F_{\text {resolved,mean }}^{\text {presure }} / F_{\text {mean }}^{\text {pressure }} \approx 1$, indicating that the VFEL method is able to resolve the buoyant forces almost exactly, as was assumed in $§ 2.2$. Therefore, we can ignore any buoyancy effects in our closures for the residual drag, and can simply focus on modeling the effect of the small-scale fluctuating stresses.

\section{Appendix A.3. Two interacting particles}

We finally seek to understand how effective our filtering approach is in resolving interparticle forces. To do so, we choose a simple test case with two particles of equal diameter in an infinite, unbounded domain. The particles are separated by a distance $r$, and their separation vector is aligned with the direction of the free stream velocity. The drag on each particle is computed using a Stokes drag law.

[50] showed that in this case, the drag on each of the particles is equal and is smaller than that predicted for a single particle in a uniform flow, and that the Stokes drag prediction is recovered as $r \rightarrow \infty$. In particular, they showed that the drag force on each particle is given by

$$
\boldsymbol{F}_{\text {drag }}^{(n)}(r)=\boldsymbol{F}_{\text {drag }}^{(n)}(r \rightarrow \infty) \lambda(r),
$$




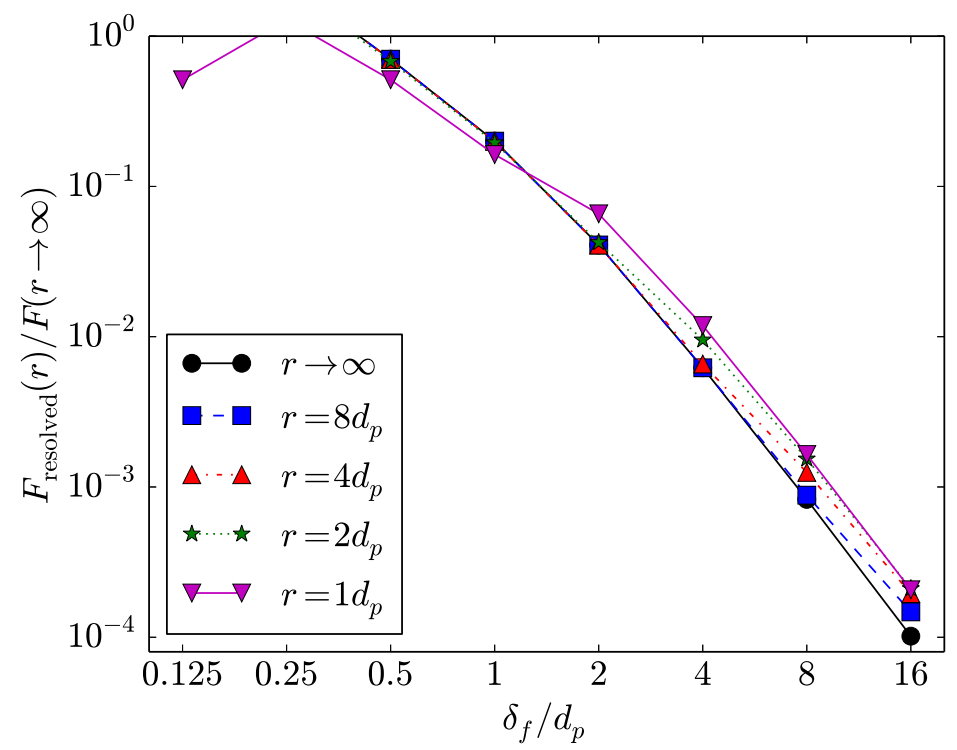

Figure A.13: The ratio of resolved to total drag for a uniform Stokes flow past two particles separated in the mean flow direction by a distance $r$. Results are shown for various filter sizes $\delta_{f}$ and are computed using (21) (which is expected to be valid in the limit of $\delta_{f} \gg d_{p}$ ).

where $\boldsymbol{F}_{\text {drag }}^{(n)}(r \rightarrow \infty)$ is the Stokes drag force for a single particle in a quiescent flow, and $\lambda(r)$ is given $b^{2}$

$$
\lambda(r) \equiv \frac{4}{3} \sinh \alpha \sum_{n=1}^{\infty}\left\{\frac{n(n+1)}{(2 n-1)(2 n+3)}\left[1-\frac{4 \sinh ^{2}[(n+1 / 2) \alpha]-(2 n+1)^{2} \sinh ^{2} \alpha}{2 \sinh [(2 n+1) \alpha]+(2 n+1) \sinh (2 \alpha)}\right]\right\},
$$

with

$$
\alpha \equiv \cosh ^{-1}\left(\frac{r}{d_{p}}\right)
$$

We therefore compute the resolved stresses $\boldsymbol{F}_{\text {resolved }}$ for this scenario. We do so using (21), which is valid for $\delta_{f} \gg d_{p}$. The results are shown in figure A.13. We notice that for $\delta_{f} / d_{p} \gtrsim 4$, we resolve a negligible portion of the overall stress, regardless of the particle separation $r$. The analytical solution given by (A11) predicts that $\lambda$ decreases with decreasing $r$ (indicating that the drag decreases as the particle separation decreases). Instead, we find that the resolved drag has the opposite trend for large $\delta_{f} / d_{p}$-it increases with decreasing separation. These changes, however, are small, and thus have a negligible effect on the particle dynamics. We therefore see that our filtering framework is unable to account for the effects of nearby particles on the particle drag force. These effects should therefore be represented using a volume-fraction-dependent drag model (e.g., see [34, 35]), which can safely be formulated without accounting for any resolved interparticle drag forces.

\footnotetext{
${ }^{2}$ The formulation given in [50] has a typographical error, as was noted by [51]. (A11) is the corrected expression.
} 


\section{Appendix B. Fluid and particle rotation in the VFEL framework}

In this section, we test the ability of the VFEL framework to capture fluid and particle rotation induced by interphase coupling. We consider two simple test cases: the torque on a sphere in a linear shear flow (Appendix B.1), and the torque induced by a rotating sphere in a quiescent fluid (Appendix B.2). We draw some practical implications of our analysis in Appendix B.3.

Appendix B.1. Torque on a sphere in linear shear flow

In Appendix A.2, we found that the VFEL framework is able to capture the forces on the particle induced by mean pressure gradients. We now test if this framework is similarly able to resolve the torque induced on particles by mean velocity gradients. To do so, we consider a single particle placed in an infinite linear shear flow. The velocity at the center of the particle is taken to be zero. The fluid velocities are given by

$$
\begin{aligned}
\frac{u_{f_{1}}}{\gamma r_{p}}= & \left(\frac{r}{r_{p}}\right) \sin \theta \sin \phi-\frac{1}{2}\left(\frac{r_{p}}{r}\right)^{4} \sin \theta \sin \phi-\frac{1}{2}\left(\frac{r_{p}}{r}\right)^{2} \sin \theta \sin \phi \\
& -\frac{5}{2}\left(\frac{r_{p}}{r}\right)^{2} \sin ^{3} \theta \cos ^{2} \phi \sin \phi+\frac{5}{2}\left(\frac{r_{p}}{r}\right)^{4} \sin ^{3} \theta \cos ^{2} \phi \sin \phi, \\
\frac{u_{f_{2}}}{\gamma r_{p}}= & -\frac{1}{2}\left(\frac{r_{p}}{r}\right)^{4} \sin \theta \cos \phi+\frac{1}{2}\left(\frac{r_{p}}{r}\right)^{2} \sin \theta \cos \phi, \\
& -\frac{5}{2}\left(\frac{r_{p}}{r}\right)^{2} \sin ^{3} \theta \cos \phi \sin ^{2} \phi+\frac{5}{2}\left(\frac{r_{p}}{r}\right)^{4} \sin ^{3} \theta \cos \phi \sin ^{2} \phi \\
\frac{u_{f_{3}}}{\gamma r_{p}}= & \frac{5}{2}\left[\left(\frac{r_{p}}{r}\right)^{4}-\left(\frac{r_{p}}{r}\right)^{2}\right] \cos \theta \sin ^{2} \theta \cos \phi \sin \phi,
\end{aligned}
$$

where $\gamma \equiv d u_{f_{1}} / d x_{2} . \quad r, r_{p}, \theta$, and $\phi$ are defined as before, but with the polar axis now aligned with $x_{3}$. The pressure is given as

$$
\frac{p}{\mu_{f} \gamma}=-5 \sin ^{2} \theta \cos \phi \sin \phi\left(\frac{r_{p}}{r}\right)^{3} .
$$

$\boldsymbol{T}^{(n)}$, the torque on particle $n$, is defined as

$$
\boldsymbol{T}^{(n)}=\int_{\mathcal{S}^{(n)}} r_{p} \hat{\boldsymbol{n}} \times(\boldsymbol{\tau} \cdot \hat{\boldsymbol{n}}) d \boldsymbol{y},
$$

where $\hat{\boldsymbol{n}}$ is the unit outward normal. As with the drag force, we divide the torque into resolved and residual components,

$$
\mathcal{T}^{(n)}=\mathcal{T}_{\text {resolved }}^{(n)}+\mathcal{T}_{\text {residual }}^{(n)}
$$

where

$$
\boldsymbol{\mathcal { T }}_{\text {resolved }}^{(n)}=\int_{\mathcal{S}^{(n)}} r_{p} \hat{\boldsymbol{n}} \times\left\{-\bar{p} \boldsymbol{I}+\mu_{f} \nabla \overline{\boldsymbol{u}_{\boldsymbol{f}}}+\mu_{f}\left(\nabla \overline{\boldsymbol{u}_{\boldsymbol{f}}}\right)^{\top}-\frac{2}{3} \mu_{f}\left(\nabla \cdot \overline{\boldsymbol{u}_{\boldsymbol{f}}}\right) \boldsymbol{I}\right\} \cdot \hat{\boldsymbol{n}} d \boldsymbol{y},
$$




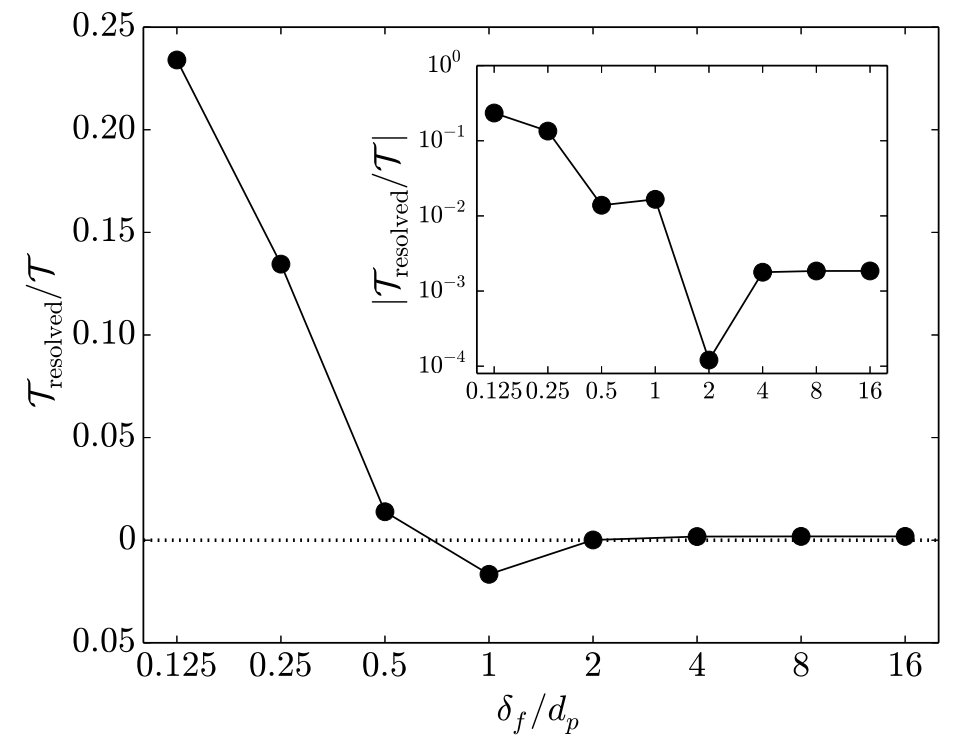

Figure B.14: The ratio of resolved to total torque for a particle in a linear shear flow for various filter sizes $\delta_{f}$. The absolute value of this ratio is shown in the inset with a logarithmic ordinate to highlight the trends at high $\delta_{f} / d_{p}$.

and

$$
\mathcal{T}_{\text {residual }}^{(n)}=\int_{\mathcal{S}^{(n)}} r_{p} \hat{\boldsymbol{n}} \times\left\{-p^{\prime} \boldsymbol{I}+\mu_{f} \nabla \boldsymbol{u}_{\boldsymbol{f}}{ }^{\prime}+\mu_{f}\left(\nabla \boldsymbol{u}_{\boldsymbol{f}}\right)^{\top}-\frac{2}{3} \mu_{f}\left(\nabla \cdot \boldsymbol{u}_{\boldsymbol{f}}{ }^{\prime}\right) \boldsymbol{I}\right\} \cdot \hat{\boldsymbol{n}} d \boldsymbol{y} .
$$

For the shear flow described above, the only non-zero torque will be along the $x_{3}$-direction, and we are considering only a single particle. We therefore use $\mathcal{T}$ (i.e., without the bold-faced notation and the superscript $n$ ) to denote the projection of the torque on a single particle along the $x_{3}$-direction. (The resolved and residual torques are defined analogously.)

We use adaptive quadrature routines provided through SciPy to compute the filtering velocities and pressures, as we did in Appendix A.1 and Appendix A.2. However, we now must sample the velocities on the surface of the particle using a full three-dimensional spherical mesh. We use 180 equally spaced grid points along the $\theta$-direction and 360 equally spaced grid points along the $\phi$-direction, giving a grid spacing of $1^{\circ}$ in each direction. As before, two grid points were used in the radial direction, with a spacing of $10^{-5} r_{p}$.

The ratio $\mathcal{T}_{\text {resolved }} / \mathcal{T}$ is plotted in figure B.14 as a function of filter size $d_{p}$, using a linear ordinate. We notice that while much of the torque is resolved at smaller values of $\delta_{f} / d_{p}$, only a small fraction is resolved when $\delta_{f} \gg d_{p}$. To emphasize this point, we have also plotted $\left|\mathcal{T}_{\text {resolved }} / \mathcal{T}\right|$ in the inset to figure B.14, using a logarithmic ordinate.

We therefore see that the VFEL is unable to capture any of the torque induced by velocity gradients for $\delta_{f} / d_{p} \gtrsim 2$ (i.e., the filter sizes typically used in VFEL studies). We next consider another canonical Stokes flow, a slowly rotating particle in a quiescent fluid, to test if the meseseale torques can be resolved in this case. 


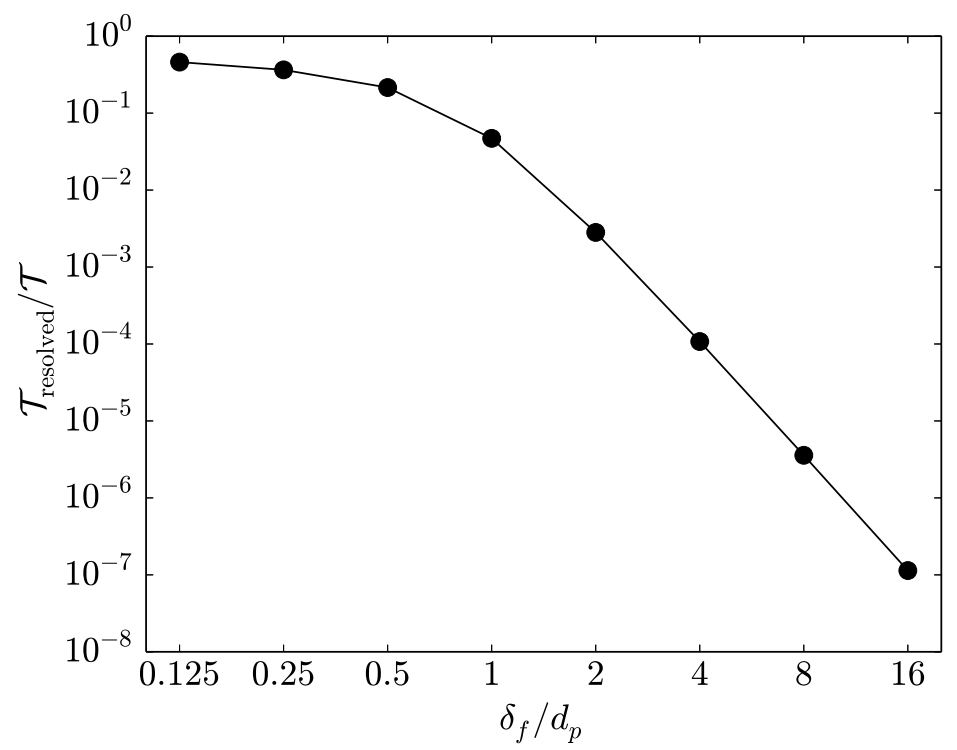

Figure B.15: The ratio of resolved to total torque for a rotating particle in infinite, initially quiescent flow for various filter sizes $\delta_{f}$.

Appendix B.2. Torque induced by a rotating sphere in a quiescent fluid

We consider a rotating sphere in a infinite, initially quiescent fluid. The steady-state velocities and pressures of the fluid in this case are given by

$$
\begin{aligned}
\frac{u_{f_{1}}}{\Omega r_{p}} & =-\left(\frac{r_{p}}{r}\right)^{2} \sin \theta \sin \phi, \\
\frac{u_{f_{2}}}{\Omega r_{p}} & =\left(\frac{r_{p}}{r}\right)^{2} \sin \theta \cos \phi, \\
\frac{u_{f_{3}}}{\Omega r_{p}} & =0
\end{aligned}
$$

and

$$
\frac{p}{\mu_{f} \Omega}=0,
$$

respectively, where $\Omega$ is the angular velocity of the sphere, and $r, \theta$, and $\phi$ are as defined in Appendix B.1.

We compute the ratio between the resolved and unresolved torque induced by the sphere, $\mathcal{T}_{\text {resolved }} / \mathcal{T}$, where $\mathcal{T}_{\text {resolved }}$ and $\mathcal{T}$ are as defined in Appendix B.1. The grid and numerical methods used in the computation of the torques are the same as in Appendix B.1. The results are plotted in figure B.15. We see that like in the case with shear, the resolved fluid velocity gradients are unable to capture the torque on the particle for moderate and large values of $\delta_{f} / d_{p}$.

Appendix B.3. Implications for simulating rotating flows with the VFEL approach

Our inability to resolve the torque on or induced by a particle is likely due to the filtering framework used in the VFEL method. In the VFEL framework, we apply volume-filtering to 
the underlying fluid velocity fields, which smooths out the fluid velocities around the particles and strongly reduces the velocity gradients. These fluid velocity gradients are responsible for the torque on the particles and the particle rotation, making us unable to capture such effects.

The VFEL framework would therefore need to be extended to allow for accurate simulations of rotating fluid-particle flows. One way to account for rotation effects could be through the addition of a microscale rotation model, in much the same way as the existing VFEL approach introduces a microscale model to account for unresolved drag forces. Such an approach, however, is beyond the scope of the current study.

\section{References}

[1] S. Sundaram, L. R. Collins, Collision statistics in an isotropic, particle-laden turbulent suspension I. Direct numerical simulations, J. Fluid Mech. 335 (1997) 75-109.

[2] J. Bec, H. Homann, S. S. Ray, Gravity-driven enhancement of heavy particle clustering in turbulent flow, Phys. Rev. Lett. 112 (2014) 184501.

[3] P. J. Ireland, A. D. Bragg, L. R. Collins, The effect of Reynolds number on inertial particle dynamics in isotropic turbulence. Part 1: Simulations without gravitational effects., J. Fluid Mech. 796 (2016) 617-658.

[4] Y. Hao, A. Prosperetti, A numerical method for three-dimensional gas-liquid flow computations, J. Comput. Phys. 196 (2004) 126-144.

[5] R. Onishi, K. Takahashi, J. C. Vassilicos, An efficient parallel simulation of interacting inertial particles in homogeneous isotropic turbulence, J. Comput. Phys. 242 (2013) 809-827.

[6] C. M. Tchen, Mean value and correlation problems connected with the motion of small particles suspended in a turbulent fluid, Ph.D. thesis, TU Delft (1947).

[7] M. R. Maxey, J. J. Riley, Equation of motion for a small rigid sphere in a nonuniform flow, Phys. Fluids 26 (1983) 883-889.

[8] R. Gatignol, The Faxén formulas for a rigid particle in an unsteady non-uniform stokesflow, J. Mec. Theor. Appl. 2 (2) (1983) 143-160.

[9] K. D. Squires, J. K. Eaton, Preferential concentration of particles by turbulence, Phys. Fluids A 3 (1991) 1169-1178.

[10] L.-P. Wang, M. R. Maxey, Settling velocity and concentration distribution of heavy particles in homogeneous isotropic turbulence, J. Fluid Mech. 256 (1993) 27-68.

[11] J. R. Fessler, J. D. Kulick, J. K. Eaton, Preferential concentration of heavy particles in a turbulent channel flow, Phys. Fluids 6 (1994) 3742-3749.

[12] L. R. Collins, A. Keswani, Reynolds number scaling of particle clustering in turbulent aerosols, New J. Phys. 6 (2004) 119. 
[13] J. P. L. C. Salazar, L. R. Collins, Inertial particle relative velocity statistics in homogeneous isotropic turbulence, J. Fluid Mech. 696 (2012) 45-66.

[14] G. H. Good, P. J. Ireland, G. P. Bewley, E. Bodenschatz, L. R. Collins, Z. Warhaft, Settling regimes of inertial particles in isotropic turbulence, J. Fluid Mech. 759 (2014) R3.

[15] B. Rosa, H. Parishani, O. Ayala, W. W. Grabowski, L. P. Wang, Kinematic and dynamic collision statistics of cloud droplets from high-resolution simulations, New J. Phys. 15 (2013) 045032.

[16] W. M. Durham, E. Climent, M. Barry, F. D. Lillo, G. Boffetta, M. Cencini, R. Stocker, Turbulence drives microscale patches of motile phytoplankton, Nat. Commun. 4 (2148) (2013) 1-7.

[17] S. Balachandar, M. R. Maxey, Methods for evaluating fluid velocities in spectral simulations of turbulence, J. Comput. Phys. 83 (1989) 96-125.

[18] S. E. Elghobashi, G. C. Truesdell, Direct simulation of particle dispersion in a decaying isotropic turbulence, J. Fluid Mech. 242 (1992) 655.

[19] P. J. Ireland, T. Vaithianathan, P. S. Sukheswalla, B. Ray, L. R. Collins, Highly parallel particle-laden flow solver for turbulence research, Comput. Fluids 76 (2013) 170-177.

[20] S. E. Elghobashi, G. C. Truesdell, On the two-way interaction between homogeneous turbulence and dispersed particles. I: Turbulence modification, Phys. Fluids A 5 (1993) $1790-1801$.

[21] P. Pepiot, O. Desjardins, Numerical analysis of the dynamics of two- and threedimensional fluidized bed reactors using an Euler-Lagrange approach, Powder Tech. 220 (2012) 104-121.

[22] J. Capecelatro, O. Desjardins, R. O. Fox, Numerical study of collisional particle dynamics in cluster-induced turbulence, J. Fluid Mech. 747 (2014) R2.

[23] J. Capecelatro, O. Desjardins, R. O. Fox, On fluid-particle dynamics in fully-developed cluster-induced turbulence, J. Fluid Mech. 780 (2015) 578-635.

[24] R. Garg, C. Narayanan, D. Lakehal, S. Subramaniam, Accurate numerical estimation of interphase momentum transfer in Lagrangian-Eulerian simulations of dispersed twophase flows, Int. J. Multiphase Flow 33 (2007) 1337-1364.

[25] P. Gualtieri, F. Picano, G. Sardina, C. M. Casciola, Exact regularized point particle method for multiphase flows in the two-way coupled regime, J. Fluid Mech. 773 (2015) $520-561$.

[26] J. A. K. Horwitz, A. Mani, Accurate calculation of stokes drag for point-particle tracking in two-way coupled flows, J. Comput. Phys. 318 (2016) 85-109. 
[27] P.-K. Yeung, S. B. Pope, An algorithm for tracking fluid particles in numerical simulations of homogeneous turbulence, J. Comput. Phys. 79 (1988) 373.

[28] M. R. Maxey, B. K. Patel, Localized force representations for particles sedimenting in stokes flow, Int. J. Multiphase Flow 27 (2001) 1603-1626.

[29] E. Climent, M. R. Maxey, Theoretical methods for micro scale viscous flows, Transworld Research Network, 2009, Ch. The force coupling method: a flexible approach for the simulation of particulate flows, pp. 1-21.

[30] K. Yeo, S. Dong, E. Climent, M. R. Maxey, Modulation of homogeneous turbulence seeded with finite size bubbles or particles, Int. J. Multiphase Flow 36 (2010) 221-233.

[31] K. Yeo, M. R. Maxey, Simulation of concentrated suspensions using the force-coupling method, J. Comput. Phys. 229 (2010) 2401-2421.

[32] J. Capecelatro, O. Desjardins, An Euler-Lagrange strategy for simulating particle-laden flows, J. Comput. Phys. 238 (2013) 1-31.

[33] S. Sundaram, L. R. Collins, A numerical study of the modulation of isotropic turbulence by suspended particles, J. Fluid Mech. 379 (1999) 105-143.

[34] R. Beetstra, M. A. van der Hoef, J. A. M. Kuipers, Drag force of intermediate reynolds number flow past mono- and bidisperse arrays of spheres, AIChE J. 53 (2007) 489-501.

[35] S. Tenneti, R. Garg, S. Subramaniam, Drag law for monodisperse gas-solid systems using particle-resolved direct numerical simulation of flow past fixed assemblies of spheres, Int. J. Multiphase Flow 37 (2011) 1072-1092.

[36] T. B. Anderson, R. Jackson, A fluid mechanical description of fluidized beds, Ind. Eng. Chem. Fundamen. 6 (1967) 527-539.

[37] L. Gibilaro, K. Gallucci, R. Di Felice, P. Pagliai, On the apparent viscosity of a fluidized bed, Chem. Eng. Sci 62 (2007) 294-300.

[38] J. Capecelatro, O. Desjardins, R. O. Fox, Effect of domain size on fluid-particle statistics in homogeneous, gravity-driven, cluster-induced turbulence, J. Fluids Eng. 138 (2015) 041301.

[39] R. H. Rangel, W. A. Sirignano, An evalulation of the point-source approximation in spray calculations, Numer. Heat Tr. A-Appl. 16 (1989) 37-57.

[40] K. Agrawal, P. N. Loezos, M. Syamlal, S. Sundaresan, The role of meso-scale structures in rapid gas-solid flows, J. Fluid Mech. 445 (2001) 151-185.

[41] M. R. Maxey, The gravitational settling of aerosol particles in homogeneous turbulence and random flow fields, J. Fluid Mech. 174 (1987) 441-465.

[42] R. A. Castleman, The resistance to the steady motion of small spheres in fluids, Tech. Notes NACA No. 231 (1926) 1-12. 
[43] R. A. Shaw, Particle-turbulence interactions in atmospheric clouds, Annu. Rev. Fluid Mech. 35 (2003) 183-227.

[44] B. J. Devenish, P. Bartello, J.-L. Brenguier, L. R. Collins, W. W. Grabowski, R. H. A. IJzermans, S. P. Malinowski, M. W. Reeks, J. C. Vassilicos, L.-P. Wang, Z. Warhaft, Droplet growth in warm turbulent clouds, Q. J. R. Meteorol. Soc. 138 (2012) 1401-1429.

[45] W. W. Grabowski, L.-P. Wang, Growth of cloud droplets in a turbulent environment, Annu. Rev. Fluid Mech. 45 (2013) 293-324.

[46] S. Dasgupta, R. Jackson, S. Sundaresan, Turbulent gas-particle flow in vertical risers, AIChE J. 40 (1994) 215.

[47] M. N. Pantzali, J. M. de Marino, G. B. Marin, G. J. Heynderickx, Three-component solids velocity measurements in the outlet section of a riser, AIChE J. 62 (2016) 35753584 .

[48] Computational and Information Systems Laboratory, Yellowstone: IBM iDataPlex System (University Community Computing), http://n2t.net/ark:/85065/d7wd3xhc (2012).

[49] I. Proudman, J. Pearson, Expansions at small Reynolds numbers for the flow past a sphere and a circular cylinder, J. Fluid Mech. 2 (1957) 237-262.

[50] M. Stimson, G. B. Jeffery, The motion of two spheres in a viscous fluid, Proc. R. Soc. Lond. A 111 (1926) 110-116.

[51] H. Faxén, The velocity of two spheres that fall under the influence of gravity in a viscous fluid, Z. angew. Math. Mech. 7 (1927) 79-80. 